\title{
Downregulation of miR-23a and miR-27a following Experimental Traumatic Brain Injury Induces Neuronal Cell Death through Activation of Proapoptotic Bcl-2 Proteins
}

\author{
Boris Sabirzhanov, ${ }^{1 *}$ Zaorui Zhao, ${ }^{1 *}$ Bogdan A. Stoica, ${ }^{1 \star}$ David J. Loane, ${ }^{1}$ Junfang Wu, ${ }^{1}$ Carlos Borroto, ${ }^{2}$ \\ Susan G. Dorsey, ${ }^{2,3}$ and Alan I. Faden ${ }^{1,3}$ \\ ${ }^{1}$ Department of Anesthesiology and Center for Shock, Trauma, and Anesthesiology Research, University of Maryland School of Medicine, ${ }^{2}$ University of \\ Maryland, School of Nursing, and ${ }^{3}$ Program in Neuroscience, University of Maryland, Baltimore, Maryland 21201
}

\begin{abstract}
MicroRNAs (miRs) are small noncoding RNAs that negatively regulate gene expression at the post-transcriptional level. To identify miRs that may regulate neuronal cell death after experimental traumatic brain injury (TBI), we profiled miR expression changes during the first several days after controlled cortical impact (CCI) in mice. miR-23a and miR-27a were rapidly downregulated in the injured cortex in the first hour after TBI. These changes coincided with increased expression of the proapoptotic Bcl-2 family members Noxa, Puma, and Bax. In an etoposide-induced in vitro model of apoptosis in primary cortical neurons, miR-23a and miR-27a were markedly downregulated as early as $1 \mathrm{~h}$ after exposure, before the upregulation of proapoptotic Bcl-2 family molecules. Administration of miR-23a and miR-27a mimics attenuated etoposide-induced changes in Noxa, Puma, and Bax, reduced downstream markers of caspase-dependent (cytochrome $c$ release and caspase activation) and caspase-independent (apoptosis-inducing factor release) pathways, and limited neuronal cell death. In contrast, miRs hairpin inhibitors enhanced etoposide-induced neuronal apoptosis and caspase activation. Importantly, administration of miR-23a and miR-27a mimics significantly reduced activation of Puma, Noxa, and Bax as well as attenuated markers of caspase-dependent and -independent apoptosis after TBI. Furthermore, miR-23a and miR-27a mimics significantly attenuated cortical lesion volume and neuronal cell loss in the hippocampus after TBI. These findings indicate that post-traumatic decreases in miR-23a and miR-27a contribute to neuronal cell death after TBI by upregulating proapoptotic Bcl-2 family members, thus providing a novel therapeutic target.
\end{abstract}

Key words: apoptosis; Bcl-2 proteins; microRNA; neuroprotection; traumatic brain injury

\section{Introduction}

Traumatic brain injury (TBI) initiates secondary cell death mechanisms that contribute to tissue damage and neurological dysfunction (Stoica and Faden, 2010). Bcl-2 homology (BH) domain 3-only proteins, such as Puma, Noxa, Bid, and Bim, promote neuronal cell death by binding and inactivating antiapoptotic Bcl-2 family members, and through direct activation of proapoptotic $\mathrm{BH}$ domain proteins (Bax and Bak), which ultimately

\footnotetext{
Received March 28, 2014; revised May 14, 2014; accepted June 16, 2014

Author contributions: B.S., B.A.S., D.J.L., and A.I.F. designed research; B.S., Z.Z., D.J.L., and J.W. performed research;B.A.S., J.W., C.B., and S.G.D. contributed unpublished reagents/analytic tools; B.S., Z.Z., B.A.S., J.W., S.G.D., and A.I.F. analyzed data; B.S., B.A.S., D.J.L., and A.I.F. wrote the paper.

This work was supported by NIH Grants R01 NS061839-05 to A.I.F. and R01 NR012686 to S.G.D. We thank Shruti Kabadi, Marie Hanscom, Marta Lipinski, and Chinmoy Sarkar for expert technical assistance.

The authors declare no competing financial interests.

*B.S., Z.Z. and B.A.S. contributed equally to this work.

Correspondence should be addressed to either of the following: Dr. Boris Sabirzhanov, Shock Trauma \& Anesthesiology Research Organized Research Center, University of Maryland School of Medicine, 655 W. Baltimore Street, Room 6-010, Baltimore, MD 21201, E-mail: bsabirzhanov@anes.umm.edu; or Dr. Bogdan A. Stoica, Department of Anesthesiology and Shock Trauma \& Anesthesiology Research Organized Research Center, School of Medicine Bressler Research Bldg, 655 W. Baltimore Street, Room 6-015, Baltimore, MD 21201, E-mail: bstoica@anes.umm.edu.

DOI:10.1523/JNEUROSCI.1260-14.2014

Copyright $\odot 2014$ the authors $\quad 0270-6474 / 14 / 3410055-17 \$ 15.00 / 0$
}

cause release of proapoptotic molecules from mitochondria (cytochrome $c$ and apoptosis-inducing factor [AIF]) (Lomonosova and Chinnadurai, 2008; Shamas-Din et al., 2011). BH3-only proteins have been implicated in neuronal cell death after CNS injury, including TBI (Engel et al., 2011). The mechanisms responsible for upregulation and activation of $\mathrm{BH} 3$-only proteins include both p53 and independent mechanisms (Jeffers et al., 2003; Yakovlev et al., 2004).

Micro-RNAs (miRs) are short (20-23 nucleotide) noncoding RNAs that negatively regulate gene expression at the posttranscriptional level by binding to the $3^{\prime}$-untranslated region (UTR) of target mRNAs, leading to their degradation and/or translational inhibition (Griffiths-Jones et al., 2006). Recent studies indicate that miRs are involved in the pathophysiology of brain seizures, ischemia, and trauma (Lei et al., 2009; Redell et al., 2009; Liu et al., 2010; Ziu et al., 2011). miRs modulate neuronal cell death pathways (Jimenez-Mateos and Henshall, 2013), but few have been directly evaluated in the context of TBI (Siegel et al., 2011; Selvamani et al., 2012), and their mechanisms of action in this regard remain largely unknown.

We performed temporal profiling of miR changes following controlled cortical impact and focused on the first hours and days 
after trauma, a period associated with maximal secondary neuronal cell death (Stoica and Faden, 2010). We hypothesized that miRs that undergo a rapid decline during this period may negatively regulate proapoptotic molecules, leading to TBI-induced activation of neuronal cell death pathways. DNA damage, including DNA breaks produced by oxidative injury and other mechanisms, is a key inducer of neuronal cell death after TBI (Clark et al., 2001). Etoposide is an anticancer drug that produces DNA breaks in neurons by inhibiting DNA-topoisomerase-II, resulting in caspase-dependent and -independent apoptosis (Pietrzak et al., 2011; Sabirzhanov et al., 2012). Here, we examined miR changes and their effects on cell death pathways after etoposideinduced DNA damage in primary neurons.

miR-23a may play an important role in regulation of apoptosis in human ovarian granulosa cells (Yang et al., 2012) and human keratinocytes (Guo et al., 2013), as well as in sex-dependent regulation of X-linked inhibitor of apoptosis (XIAP) after cerebral ischemia (Siegel et al., 2011). Previous studies that examined miR modulation after TBI have been largely descriptive and have focused only tangentially on the miR-23a 27a 24-2 cluster (Lei et al., 2009; Truettner et al., 2011; Hu et al., 2012). In this study, we identified miR-23a and miR-27a from an miR array because they were downregulated in the acute time period after TBI that is associated with neuronal cell death; they are members of the same genomic cluster that are expressed together as single primary transcript; and they are predicted to target members of the proapoptotic $\mathrm{Bcl} 2$ family.

\section{Materials and Methods}

Animals. Studies were performed using young adult (3-month-old, 22-26 g) male C57BL/6 mice, which were housed under a $12 \mathrm{~h}$ light-dark cycle, with ad libitum access to food and water. All surgical procedures complied with the Guide for the Care and Use of Laboratory Animals published by the National Institutes of Health (DHEW publication NIH 85-23-2985), and the protocols were approved by the University of Maryland School of Medicine Institutional Animal Care and Use Committee.

Controlled cortical impact (CCI) injury. Our custom-designed CCI injury device (Fox et al., 1998) consists of a microprocessor-controlled pneumatic impactor with a $3.5-\mathrm{mm}$-diameter tip. Young adult male $\mathrm{C} 57 \mathrm{BL} / 6$ mice were anesthetized with isoflurane evaporated in a gas mixture containing $70 \% \mathrm{~N}_{2} \mathrm{O}$ and $30 \% \mathrm{O}_{2}$ and administered through a nose mask (induction at $4 \%$ and maintenance at $2 \%$ ). Depth of anesthesia was assessed by monitoring respiration rate and pedal withdrawal reflexes. Mice were placed on a heated pad, and core body temperature was maintained at $37^{\circ} \mathrm{C}$. The head was mounted in a stereotaxic frame, and the surgical site was clipped and cleaned with Nolvasan and ethanol scrubs. A $10 \mathrm{~mm}$ midline incision was made over the skull, the skin and fascia were reflected, and a $4 \mathrm{~mm}$ craniotomy was made on the central aspect of the left parietal bone. The impounder tip of the injury device was then extended to its full stroke distance $(44 \mathrm{~mm})$, positioned to the surface of the exposed dura, and reset to impact the cortical surface. Moderate-level injury was induced using an impactor velocity of $6 \mathrm{~m} / \mathrm{s}$ and deformation depth of $2 \mathrm{~mm}$ as previously described (Loane et al., 2009). After injury, the incision was closed with interrupted 6-0 silk sutures, anesthesia was terminated, and the animal was placed into a heated cage to maintain normal core temperature for $45 \mathrm{~min}$ after injury. All animals were monitored for at least $4 \mathrm{~h}$ after surgery and then daily. Sham-injured animals underwent anesthesia and surgical procedures but without cortical impact.

Intracerebroventricular injections. At 15 min after injury, mice received a single intracerebroventricular injection of miR-23a-3p, miR-27a-3p, or negative control miR mimics. All drugs were made up in aCSF, and drugs were injected into the left ventricle (coordinates from bregma $=$ anterior,
-0.5 ; lateral, -1.0 ; ventral, -2.0 ) using a 30 gauge needle attached to a Hamilton syringe at a rate of $0.5 \mathrm{ml} / \mathrm{min}$, with a final volume of $5 \mu \mathrm{l}$ of 0.1 mM miR mimic solution.

Cell cultures. Rat cortical neurons (RCNs) were derived from rat embryonic cortices. Cells were seeded onto poly-D-lysine-coated 96 well or 24 well plates or $100 \mathrm{~mm}$ Petri dishes (cell density $1 \times 10^{6} / \mathrm{cm}^{2}$ ) and maintained in serum-free conditions using the B27 supplement as described previously (Yakovlev et al., 2001). Transfection of RCNs was performed at $6 \mathrm{~d}$ in vitro (DIV). RCNs were transfected with miR mimics and hairpin inhibitors using the Lipofectamine RNAiMAX Transfection Reagent, Lipofectamine 2000 (Invitrogen) used for RCN cotransfection with plasmid and miR mimics according to the manufacturer's protocol. The concentrations of miRs mimics and hairpin inhibitors commonly used for transfection are in the range 10-100 nM (Stappert et al., 2013; Yan et al., 2013). Based on preliminary titration experiments, we chose a final concentration of $50 \mathrm{~nm}$ for the miR mimics and hairpin inhibitors.

This concentration resulted in optimal transfection efficiency devoid of nonspecific changes in nontargeted miRs and no neurotoxic effects. Moreover, the chosen concentration was associated with peak neuroprotective effects (data not shown). Four hours after transfection, media was replaced with normal condition media and cells treated with etoposide at a final concentration $50 \mu \mathrm{M}$. The following miR mimics and hairpin inhibitors were used: miRIDIAN microRNA Mimic Negative Control (-ve con mimic) (CN-001000-01-05); miRIDIAN Mimic, Rat rno-miR23a-3p (C-320309-03-0005); miRIDIAN Mimic, Rat rno-miR-27a-3p (C-320317-03-0005); miRIDIAN microRNA Hairpin Inhibitor Negative Control (-ve con inhibit) (IN-001005-01-05); miRIDIAN microRNA Rat rno-miR-23a-3p Hairpin Inhibitor (IH-320309-05-0005); miRIDIAN microRNA Rat rno-miR-27a-3p Hairpin Inhibitor (IH-320317-05-0005) (Thermo Scientific). Sequences of both miRIDIAN microRNA Mimic Negative Control and miRIDIAN microRNA Hairpin Inhibitor Negative Control are based on Caenorhabditis elegans microRNAs and have minimal sequence identity in human, mouse, and rat.

The human neuroblastoma SH-SY5Y cells were seeded in 96-well plates and maintained in DMEM (Invitrogen) supplemented with 10\% FCS, $100 \mathrm{U} / \mathrm{ml}$ penicillin, and $100 \mathrm{U} / \mathrm{ml}$ streptomycin in a humid atmosphere of $5 \% \mathrm{CO}_{2}$ and $95 \%$ air at $37^{\circ} \mathrm{C}$. Cells at $70 \%$ confluence were transfected with plasmids. Transfection of cells was performed by using Lipofectamine 2000 Reagent (Invitrogen) according to the manufacturer's protocol.

Cell death, cell viability, and in-plate fluorometric caspase-3 assays. Cell death, cell viability, and in-plate fluorometric caspase-3 activity were measured as previously described using the $\mathrm{LDH}$, Calcein AM, and DEVD-AMC assays, respectively (Stoica et al., 2003) on BioTek Synergy Ht Microplate Reader (BioTek). Each individual treatment/time point reflects six replicates for all assays performed on cortical neurons cultured in 96-well plates; all wells were plated with and contained the same number of cells.

Construction of reporter plasmids and Luciferase assays. Oligonucleotide pairs were annealed, ligated, and cloned into pmirGLO vector (Promega) digested with XbaI and SalI according to the manufacturer's protocol. Oligonucleotides matched to the $21 \mathrm{bp} \mathrm{mmu}-$ miR-23a-3p and miR-27a-3p target sequence were used to produce pmiR-23aGlo and pmiR-27aGlo plasmids. Internal restriction site for NotI were added in flanking sequences of oligonucleotides for clone confirmation. To produce reporter plasmids containing $3^{\prime}$ UTRs of mouse PUMA, Noxa, and Bax, sequences were PCR-amplified, digested, gel purified, ligated, and cloned into pmirGLO vector (Promega) digested with XbaI and SalI restriction enzymes. The following primers were used to amplify $3^{\prime}$ UTR of mouse PUMA, Noxa, and Bax: PUMA 3'UTR forward primer 5' reverse primer $5^{\prime}$-tgGTCGACCACTGTTCAATCTGATTTTATTGA AAAGGA-3'; Noxa $3^{\prime}$ UTR forward primer $5^{\prime}$-tg TCTAGAGTTCTTC CAAAGCTTTTGCA- $3^{\prime}$, reverse primer $5^{\prime}$-tgGTCGACGCATTTTTCAA TAGTTACTTTAGTATCAAC-3'; Bax 3' UTR forward primer 5' -tgTCTA GAGGCCTCCCACTGCCTTGG, reverse primer $5^{\prime}$-tgGTCGACTACAA TCCAAAGTGGACCTGAGG-3' ${ }^{\prime}$. XbaI site was added on $5^{\prime}$ end of forward primers, and SalI site was added to $5^{\prime}$ end of reverse primers for cloning into pmirGLO vector XbaI, SalI digested vector (XbaI and SalI 
A

\begin{tabular}{|c|c|c|c|c|c|}
\hline miRNA & \multicolumn{4}{|c|}{ Fold change } & p-value \\
\hline & $\mathbf{1 h}$ & $\mathbf{6 h}$ & $\mathbf{2 4 h}$ & $\mathbf{7 2 h}$ & \\
\hline miR-23a & 0.44 & 0.43 & 0.42 & 1.16 & 0.0003 \\
\hline miR-27a & 0.45 & 0.49 & 0.46 & 1.30 & 0.0225 \\
\hline
\end{tabular}

B

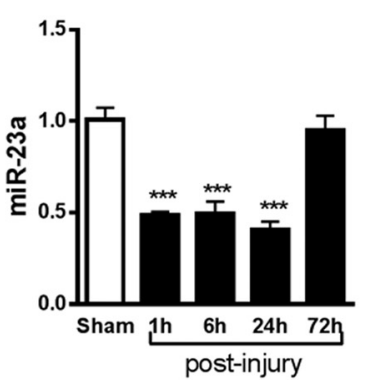

C
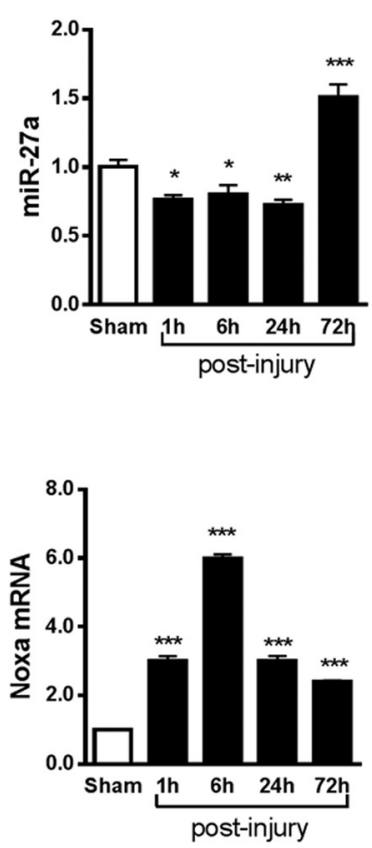

Figure 1. miR-24-3p and miR-27a-3p downregulation in the mouse-injured cortex after TBI is associated with increased mRNA levels for Bcl-2 family proapoptotic molecules. $A$, Analysis of gene array data for miR-23a-3p and miR-27a-3p expression at different time points after TBI in mouse cortex after TBI. Data are fold change compared with uninjured controls. We performed an ANOVA for each miR over time compared with uninjured controls. The $p$ values, which were adjusted for multiple testing correction, indicate overall significance of the ANOVA. $\boldsymbol{B}$, Analysis of $q P C R$ data for miR-23a-3p and miR-27a-3p expression at different time points after TBI in mouse cortex after TBI, normalized to snoRNA202. Data are mean \pm SD. ${ }^{*} p<0.05$ versus control animals $(N=5)$. ${ }^{* *} p<$ 0.01 versus control animals $(N=5) .{ }^{* * *} p<0.001$ versus control animals $(N=5) . C, q P C R$ quantification of expression of proapoptotic genes Noxa and Puma in mouse cortex at different time points after TBI, normalized to GAPDH. Data are mean \pm SD. ${ }^{*} p<0.05$ versus sham animals $(N=5) .{ }^{* *} p<0.01$ versus sham animals $(N=5) .{ }^{* * *} p<0.001$ versus sham animals $(N=5)$. Analysis by one-way ANOVA followed by multiple pairwise comparisons using Student-Newman-Keuls post hoc test.

sites are marked bold). RCNs or SH-SY5Y was cultured in 96-well plates and transfected as described above. All assays were performed at $24 \mathrm{~h}$ after transfection with the dual Luciferase assay (Promega) on BioTek Synergy Ht Microplate Reader (BioTek). Firefly Luciferase activity was normalized to Renilla Luciferase activity. Experiments were performed in triplicate.

RNA isolation. Total RNA was isolated using miRNeasy Kit (QIAGEN). During the process of isolation, samples were treated with RNase-free DNase (QIAGEN) to digest DNA contamination of the samples according to the manufacturer's protocol.

miRNA arrays and miRNA array data analysis. A total of $100 \mathrm{ng}$ of total RNA was used for miR expression profiling using Affymetrix GeneChip miRNA 2.0 Arrays (Affymetrix). Briefly, total RNA from cells was extracted as described above. RNA quality and quantity were assessed via spectrophotometer (Nanodrop 1000; Thermo Scientific) and also via analysis of RNA Integrity Number using the Agilent Bioanalyzer (Agilent Technologies). Double stranded cDNA was prepared from RNA and used as the template for in vitro transcription to prepare biotinylated cRNA. The target was fragmented and hybridized to probes using standard Affymetrix protocols. Affymetrix's miRNA QC Tool was used to evaluate quality control on all image data. Expression values were computed using normalization function RMA (Irizarry et al., 2003) from
Bioconductor's affy (Gautier et al., 2004) package. Only values annotated as mouse probes $(\mathrm{Mmu})$ were further considered. Expression values for the dataset were fitted to a linear model to determine differentially expressed miRNAs between different time points. Bioconductor's package LIMMA (Gentleman, 2005) was used for this task. Comparisons were made between the control group (time $0 \mathrm{~h}$ ) and each of the injured groups (times 1, 6, 24, and $72 \mathrm{~h} ; n=3$ arrays per group/time). Those miRNAs showing an adjusted $p$ value (false discovery rate $<0.05$ for any of these comparisons) were determined to be differentially expressed and selected for further analysis.

$q P C R$. Verso cDNA Kit (Thermo Scientific) was used to synthesize cDNA from purified total RNA. RNA $(1 \mu \mathrm{g})$ was heated to $70^{\circ} \mathrm{C}$ for $5 \mathrm{~min}$ and mixed with $5 \times$ cDNA-synthesis buffer, dNTP mix ( $0.5 \mathrm{~nm}$ final concentration), and Verso Enzyme Mix, and finally random hexamers ( $400 \mathrm{ng} / \mu \mathrm{l}$ ) were added. Tubes were incubated at $42^{\circ} \mathrm{C}$ for $30 \mathrm{~min}$, followed by $95^{\circ} \mathrm{C}$ for $2 \mathrm{~min}$. Quantitative real-time PCR amplification was performed by using cDNA TaqMan Universal Master Mix II (Applied Biosystems). In brief, reactions were performed in duplicate containing $2 \times$ TaqMan Universal Master Mix II, $1 \mu$ l of cDNA (corresponding to 50 ng RNA/reaction), and TaqMan Gene Expression Assay (Applied Biosystems), $20 \times$ in a final volume of $20 \mu$ l. TaqMan Gene Expression assays for following mouse genes were performed: GAPDH (Mm99999915_g1), 


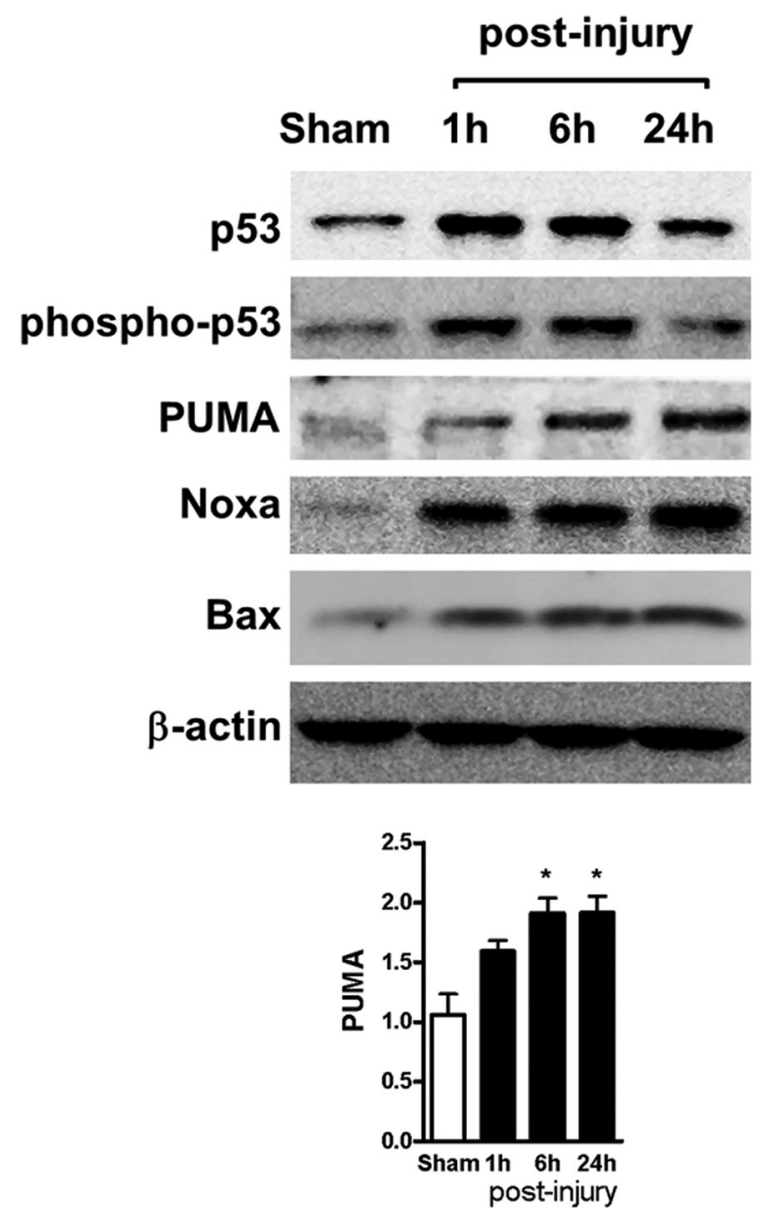

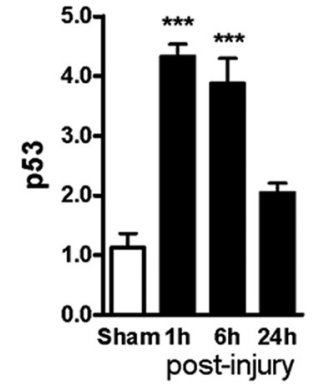
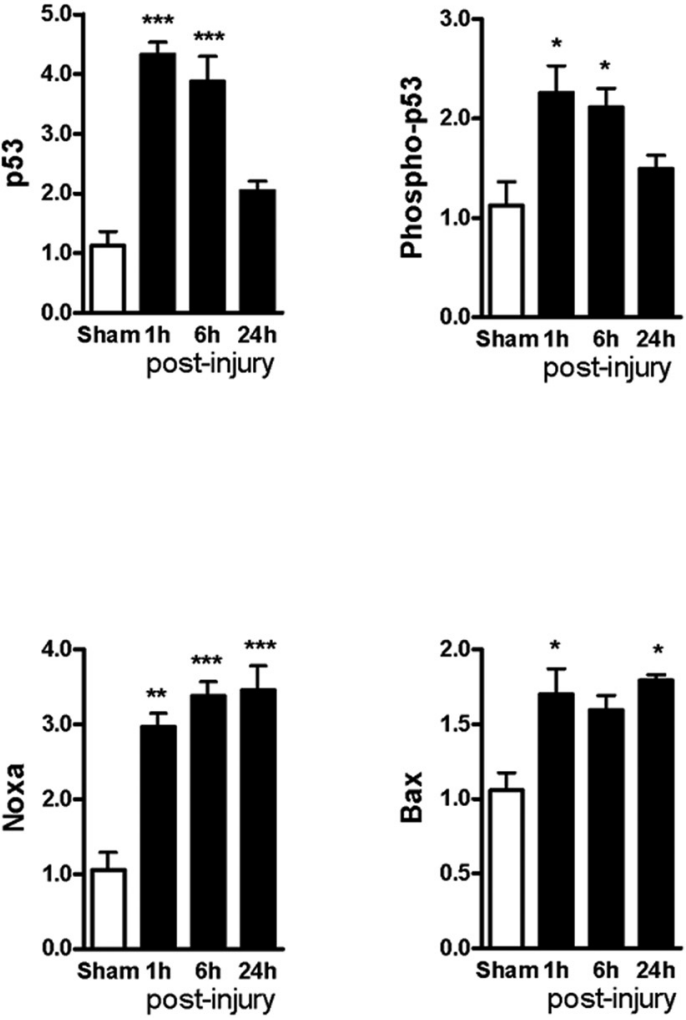

Figure 2. Activation of $\mathrm{p} 53$ and increased protein expression of proapoptotic B $\mathrm{C} 12$ molecules after TBI. Western blot analysis of the level of proapoptotic proteins in mouse-injured cortex after TBI. Tissue lysates were fractioned on SDS-polyacrylamide gel and immunoblotted with antibodies against p53, phosphorylated p53 (Ser 15), PUMA, Noxa, Bax, and $\beta$-actin. Levels of p53, phosphorylated p53 (Ser 15), PUMA, Noxa, and Bax were quantified as fold change to control levels after measurement of band intensity by densitometry and normalization to levels of $\beta$-actin. Data are mean $\pm S D .{ }^{*} p<0.05$ versus sham $(N=4) .{ }^{* *} p<0.01$ versus sham $(N=4) .{ }^{* *} p<0.001$ versus sham $(N=4)$. Analysis by one-way ANOVA followed by multiple pairwise comparisons using Student-Newman-Keuls post hoc test.

Noxa (Mm00451763_m1), PUMA (Mm00519268_m1), Bim (Mm00437796_m1), Bak1 (Mm00432045_m1), Xiap (Mm00776505_m1), Map4k4 (Mm00500812_m1), Map2k7 (Mm00488765_g1); Calpain-6 (Mm00500361_m1), and Calpain-7 (Mm00486697_m1); for rat: GAPDH (Rn01775763_g1), Noxa (Rn01494552_m1), Puma (Rn00597992_m1), Bim (Rn00674175_m1), Bak1 (Rn01429084_m1), Xiap (Rn01457299_m1), Bax (Rn02532082_g1), Map4k4 (Rn01437980_m1), Map2k7 (Rn01403106_ m1), Calpain-6(Rn00582574_m1), and Calpain-7 (Rn01453530_m1) (Applied Biosystems). Reactions were amplified and quantified using a 7900HT Fast Real-Time PCR System and the corresponding software (Applied Biosystems). The PCR profile consisted of one cycle at $50^{\circ} \mathrm{C}$ for $2 \mathrm{~min}$ and $95^{\circ} \mathrm{C}$ for $10 \mathrm{~min}$, followed by 40 cycles at $95^{\circ} \mathrm{C}$ for $15 \mathrm{~s}$ and $60^{\circ} \mathrm{C}$ for $1 \mathrm{~min}$. All reactions were performed twice. Efficiency of reactions for each set gene expression was close to $100 \%$. Efficiency of reactions was measured using the $\mathrm{C}_{\mathrm{T}}$ slope method. Briefly, serial dilutions of samples were generated, and real-time RT-PCRs were performed on each dilution. The $\mathrm{C}_{\mathrm{T}}$ values were then plotted versus the log of the dilution, and a linear regression was performed. Efficiency $=(10-1 /$ slope 1) $\times 100 \%$ (Pfaffl, 2001). Samples were confirmed to be free of DNA contamination by performing reactions without reverse transcriptase. Gene expression was normalized to GAPDH, and the relative quantity of mRNAs was calculated based on the comparative $C_{T}$ method (Livak and Schmittgen, 2001).

$m i R$ reverse transcription. Quantitative real-time PCR was used to measure expression of individual miR-23a and miR-27a. A total of $10 \mathrm{ng}$ of total RNA was reverse transcribed using TaqMan miRNA Reverse Transcription Kit (Applied Biosystems) with miRNA-specific primers. Re- verse transcription reaction products $(1.5 \mu \mathrm{l})$ were used for $\mathrm{qPCR}$ as described above. TaqMan Gene Expression assays for the following miRs were used: miR-23a (000399); miR-27a (000408); miR-24 (000402); miR-23b (000400); miR-27b (000409); U6 snRNA (001973); and snoRNA202 (001232) (Applied Biosystems).

Antibodies. Antibodies from different vendors were used in this study. Abcam: V5 (ab27674); Histone H2A.X (ab11175); Santa Cruz Biotechnology: AIF (sc-13116); Apaf-1 (sc-65890); cytochrome $c$ (sc-13560); FAS (sc-716); Bim (sc-11425); Cell Signaling Technology: Cleaved Caspase-3 (\#9661); Cleaved PARP (\#9545); Phospho-Histone H2A.X (Ser139) (\#9718); Phospho-p53 (Ser15) (\#9284); p53 (1C12) (\#2524); Bax (\#2772); XIAP (\#2042); Enzo Life Sciences: GAPDH (ADI-CSA335); Bax (active monomer) (ALX-804-224-C100); $\alpha$-fodrin (BMLFG6090); ProSci: PUMA (\#3041); Noxa (\#2437); EMD Millipore: Bak (06-536); Sigma: $\beta$-actin (A1978).

Cell lysates preparation and Western blot. Whole-cell extracts were prepared as described previously (Stoica et al., 2005). A portion of the lysate (20 $\mu \mathrm{g}$ of protein) was then fractionated by SDS-PAGE. Western blot was performed as described previously (Stoica et al., 2005). Membranes were washed and protein complexes were visualized using SuperSignal West Dura Extended Duration Substrate (Pierce). Chemiluminescence was captured on a Kodak Image Station 4000R station (Carestream Health), and protein bands were quantified by densitometric analysis using Molecular Imaging Software (Carestream Health). The images were acquired under conditions that did not cause saturation of the signal. The data presented reflect the intensity of the target protein band compared 
A

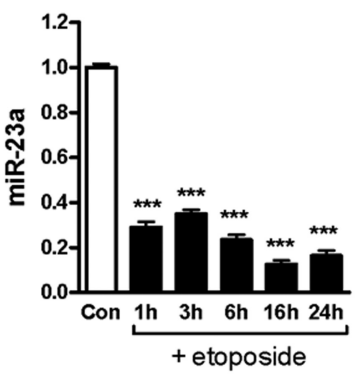

B
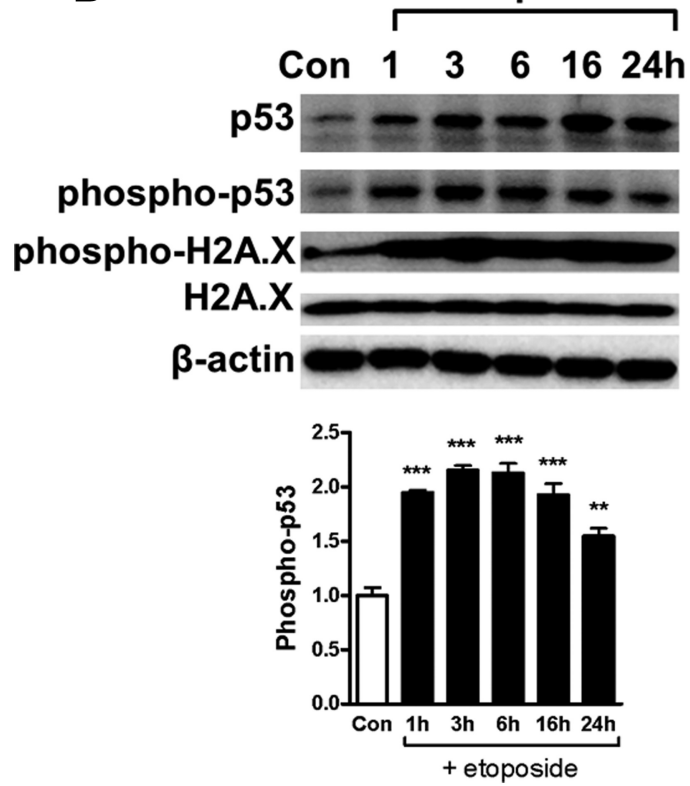
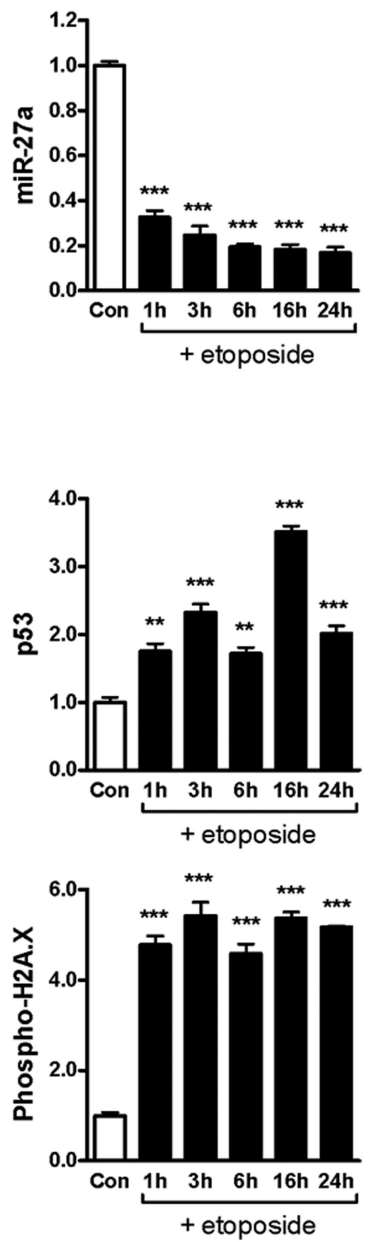

Figure 3. miR-24-3p and miR-27a-3p downregulation in an in vitro model of etoposide-induced neuronal apoptosis is associated with DNA damage, and increased expression and activation of $\mathrm{p} 53$ in primary cortical neurons. $A$, qPCR quantification of miR-23a-3p and miR-27a-3p expressions in RCNs at different time points after etoposide treatment. Levels of miRs were normalized to U6 snRNA. Neuronal apoptosis was induced by etoposide as described above. Data are mean $\pm S D .{ }^{*} p<0.05$ versus control untreated $\mathrm{RCNs}(N=4) .{ }^{* *} p<0.01$ versus control untreated $\mathrm{RCNs}(N=4) .{ }^{* *} p<0.001$ versus control untreated $\mathrm{RCNs}(N=$ 4). $\boldsymbol{B}$, Western blot analysis of the level of $\mathrm{p} 53$ and phosphorylated $\mathrm{p} 53$ in primary cortical neurons at different time points after etoposide treatment. Cell lysates were fractioned on SDS-polyacrylamide gel and immunoblotted with antibodies against p53, phosphorylated p53 (Ser 15), histone H2A.X, phosphorylated histone H2A.X (Ser 139), and $\beta$-actin. Levels of p53, phosphorylated p53 (Ser 15), and phosphorylated histone H2A.X (Ser 139) were quantified as fold change to control levels after measurement of band intensity by densitometry and normalization to levels of $\beta$-actin. Data are mean $\pm S D .{ }^{*} p<0.05$ versus control untreated $\operatorname{RCNs}(N=4) .{ }^{* *} p<0.01$ versus control untreated RCNs $(N=4) .{ }^{* *} p<0.001$ versus control untreated $\operatorname{RCNs}(N=4)$. Analysis by one-way ANOVA followed by multiple pairwise comparisons using Student-Newman post hoc test.

with the control and were normalized based on the intensity of the endogenous control for each sample (expressed in arbitrary units).

Subcellular fractionation. Subcellular fractionation was performed as described previously (Stoica et al., 2005) with some modifications. RCNs were harvested and washed in ice-cold PBS. Cell suspension was centrifuged at $500 \times g$ for $15 \mathrm{~min}$ at $4^{\circ} \mathrm{C}$. Cell pellet was resuspended for $10 \mathrm{~min}$ on ice in the digitonin lysis buffer (20 mM HEPES, pH 7.4, $80 \mathrm{~mm} \mathrm{KCl,} 1$ mM EDTA, 1 mM EGTA, 1 mM DTT, 250 mm sucrose, $200 \mu \mathrm{g} / \mathrm{ml}$ digitonin, and protease inhibitor and phosphatase inhibitor cocktails (P8340; P5726 Sigma-Aldrich). The lysate was centrifuged at $1000 \times g$ for $5 \mathrm{~min}$ at $4^{\circ} \mathrm{C}$ to pellet the nuclei. The supernatant was transferred to a new tube and centrifuged again at $12000 \times g$ for $10 \mathrm{~min}$ at $4^{\circ} \mathrm{C}$ to pellet the mitochondria. The resulting supernatant, representing the cytosolic fraction, was recovered. Nuclear and mitochondrial lysates were prepared in RIPA buffer (Teknova) with protease inhibitor cocktail (P8340 Sigma-Aldrich).

Lesion volume assessment. Mice were killed and transcardially perfused with saline and $10 \%$ buffered formalin phosphate solution (containing
4\% PFA; Fisher Scientific) on postinjury day 28. Lesion volume was determined based on the Cavalieri method as previously described (Kabadi et al., 2012) using Stereoinvestigator software (MBF Biosciences).

Assessment of neuronal cell loss in hippocampal subregions. Stereoinvestigator software (MBF Biosciences) was used to count the total number of surviving neurons in the Cornu Ammonis (CA) $1, \mathrm{CA} 2 / 3$, and dentate gyrus (DG) subregions of the hippocampus using the optical fractionator method of stereology as previously described (Kabadi et al., 2012).

Statistical analysis. Analysis was performed using the Sigmaplot Software (version 12). If the data passed a normality test, further analysis involved one-way ANOVA followed by multiple pairwise comparisons using StudentNewman-Keuls post hoc test. If the data failed the normality test, further analysis involved nonparametric Kruskal-Wallis one-way ANOVA followed by multiple pairwise comparisons using Dunn's post hoc test.

\section{Results}

miR-24-3p and miR-27a-3p are downregulated and Bcl-2 family proapoptotic molecules are upregulated in the injured cortex after TBI

We profiled miRs expression by $\mathrm{Ge}$ neChip miRNA array in the cortex of CCI mice. These data indicated that TBI significantly downregulated miR-23a and miR$27 \mathrm{a}$, members of the miR-23a $27 \mathrm{a} \sim 24-2$ cluster (Fig. 1A). To confirm these changes, we performed a detailed expression profile analysis of the members of this cluster using qPCR and observed a rapid downregulation of miR-23a-3p and miR-27a-3p starting as early as $1 \mathrm{~h}$ after injury and lasting up to $24 \mathrm{~h}$, followed by recovery at $72 \mathrm{~h}$ after injury (Fig. $1 B$ ). In contrast, qPCR analysis showed no significant changes after TBI in the expression profiles of miR-24, the other member of the miR-23a $\sim 27 \mathrm{a} \sim 24-2$ cluster, or in the expression of the paralog cluster miR23b $27 \mathrm{~b} \sim 24-1$ (data not shown).

The mRNA expression levels for various Bcl-2 family proapoptotic proteins were analyzed in the cortex at different time points after TBI. qPCR demonstrated rapid upregulation of the $\mathrm{BH} 3$-only family members Noxa (Fig. 1C), Bid and Bim (data not shown), with peak levels at $6 \mathrm{~h}$ after injury, followed by progressive decrease toward normal levels at $24 \mathrm{~h}$ after injury. PUMA mRNA reached its peak at $1 \mathrm{~h}$ after injury (Fig. 1C), and Bak mRNA (a proapoptotic multi-BH domain member) reached its peak at $1 \mathrm{~h}$ after injury and remained elevated thereafter (data not shown).

The mRNA data were corroborated by quantitative determination of protein levels of several key proapoptotic proteins using Western blot. The protein levels of total and phosphorylated (activated) p53, a key regulator of proapoptotic Bcl2 family (Vousden, 2005), increased rapidly and reached their peak at $1 \mathrm{~h}$ after injury and decreased toward control levels at $24 \mathrm{~h}$ after injury (Fig. 2). The protein levels of BH3-only proteins Noxa and Puma 
A
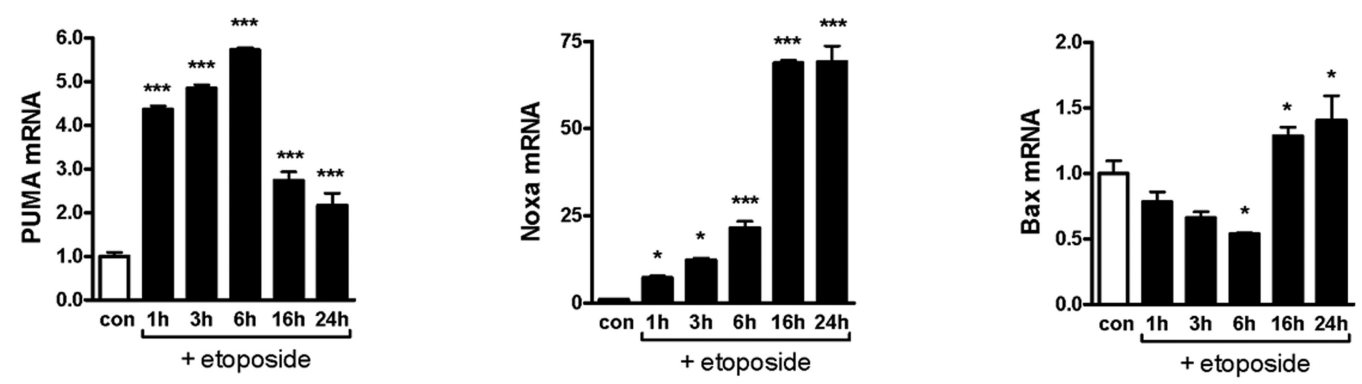

B
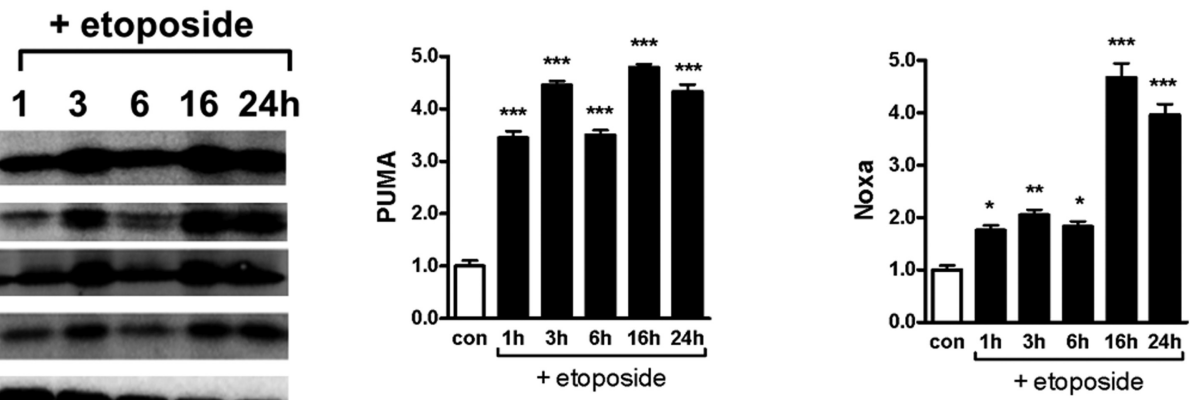

\section{$\beta$-actin}
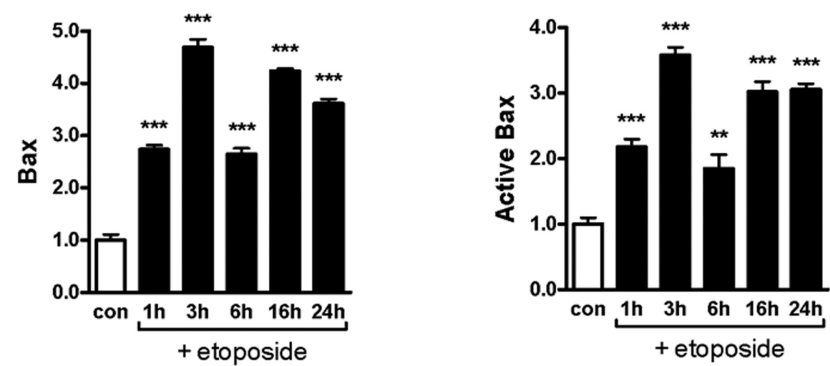

Figure 4. $\mathrm{mRNA}$ and protein levels of proapoptotic members of $\mathrm{BCl}-2$ family were increased in etoposide-treated primary cortical neurons. $A, q \mathrm{PCR}$ quantification of expression of proapoptotic genes: Noxa, Puma, and Bax in primary cortical neurons at different time points after etoposide treatment. Level of gene expression was normalized to GAPDH expression. Neuronal apoptosis were induced by etoposide as described above. Data are mean $\pm S D$. ${ }^{*} p<0.05$ versus control untreated $R C N s(N=4) .{ }^{* *} p<0.01$ versus control untreated $R C N s(N=4)$. ${ }^{* * *} p<0.001$ versus control untreated RCNs $(N=4)$. $\boldsymbol{B}$, Western blot analysis of the level of proapoptotic proteins in primary cortical neurons at different time points after etoposide treatment. Cell lysates were fractioned on SDS-polyacrylamide gel and immunoblotted with antibodies against PUMA, Noxa, Bax, active Bax, and $\beta$-actin. Levels of PUMA, Noxa, Bax, and active Bax were quantified as fold change to control levels after measurement of band intensity by densitometry and normalization to levels of $\beta$-actin. Data are mean \pm SD. ${ }^{*} p<0.05$ versus control untreated $\mathrm{RCNs}(N=4)$. ${ }^{* *} p<0.01$ versus control untreated RCNs $(N=4) .{ }^{* * *} p<0.001$ versus control untreated RCNs $(N=4)$. Analysis by one-way ANOVA followed by multiple pairwise comparisons using Student-Newman-Keuls post hoc test.

reached their peak at $6 \mathrm{~h}$ after injury and remained elevated at $24 \mathrm{~h}$ after injury (Fig. 2). The protein levels of the proapoptotic multi-BH domain protein Bax were significantly increased as early as $1 \mathrm{~h}$ after injury and remained elevated through $24 \mathrm{~h}$ after injury (Fig. 2).

We did not observe any significant changes in XIAP expression at mRNA or protein level after TBI in male mice (data not shown). In addition, qPCR analysis demonstrated that expression of some of predicted miR-23a-3p and miR-27a-3p targets involved in apoptosis pathways, such as MAP4K4, MAP2K7, calpain-6 and -7, was downregulated after TBI (data not shown).

miR-24-3p and miR-27a-3p are downregulated and p53 is activated in an in vitro model of etoposide-induced neuronal apoptosis

To explore the putative mechanisms of miR regulation of cell death, we used an in vitro model of etoposide-induced primary cortical neuron apoptosis (Sabirzhanov et al., 2012). Levels of miR-24-3p and miR-27a-3p were analyzed by qPCR at different time points after etoposide treatment. Our data demonstrate significant reduction in levels of both miR-24-3p and miR-27a-3p compared with control. Both miRs were downregulated rapidly, as early as $1 \mathrm{~h}$ after etoposide treatment (Fig. 3A). Quantitative Western blot analysis demonstrated a rapid $(1 \mathrm{~h})$ and sustained elevation in p53 expression and p53 phosphorylation on Ser 15 (markers of activation of the $\mathrm{p} 53$ pathways), as well as phosphorylated histone H2A.X (Ser 139), a marker of DNA damage (Fig. $3 B$ ). Similar to the TBI data, we did not observe changes in expression of miR-24 or in the expression of the paralog cluster miR-23b 27b 24-1 after etoposide treatment (data not shown). The expression of miR-23-3p and miR-27a-3p in primary microglia and astrocytes was at least 100 times smaller compared with primary neurons (data not shown).

Proapoptotic members of Bcl-2 family are upregulated in etoposide-treated primary cortical neurons

Some of the most important pathways induced by p53 activation involve proapoptotic members of Bcl-2 family. qPCR analysis demonstrated increased gene expression of proapop- 


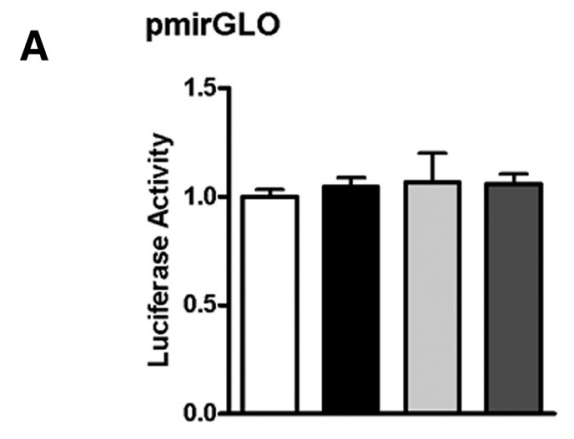

pmiR-23aGLO

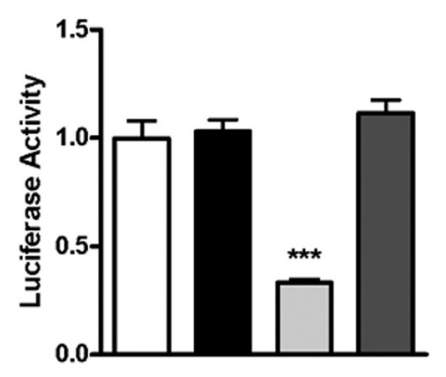

pmiR-27aGLO
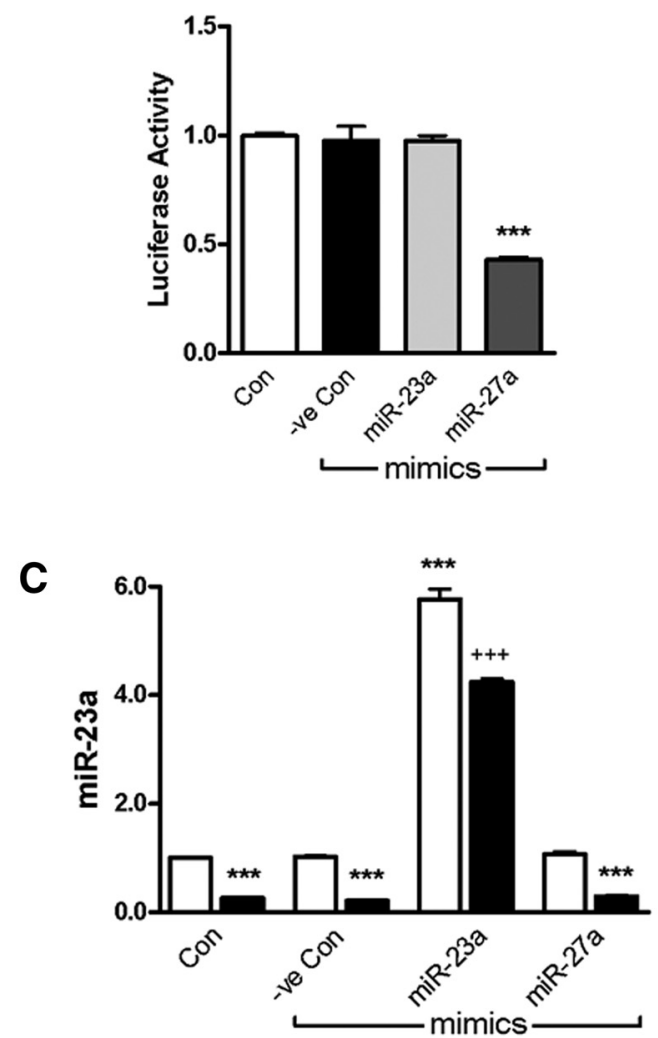
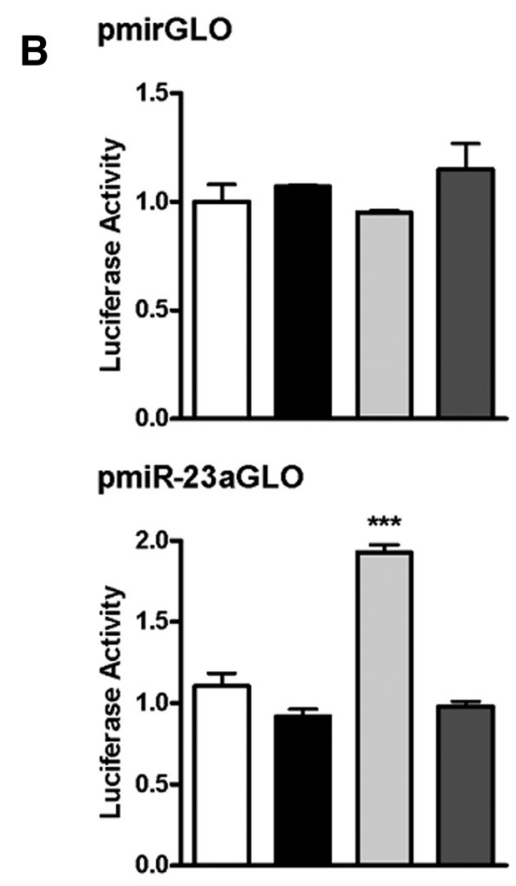

pmiR-27aGLO
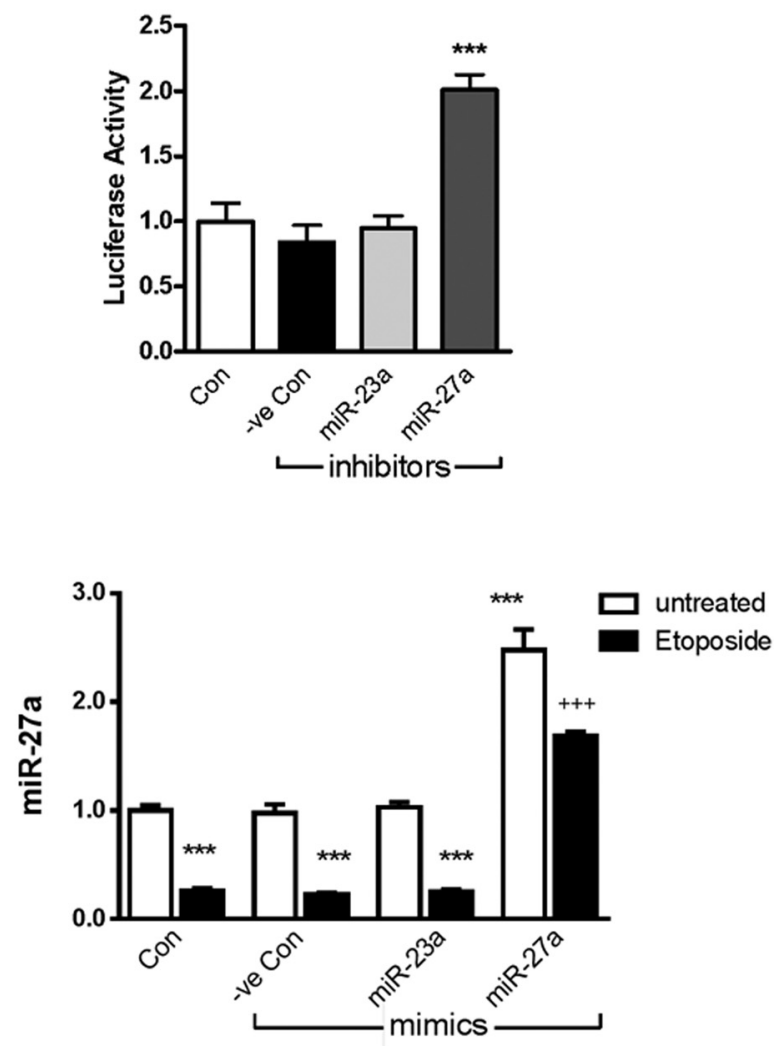

Figure 5. miR-23a-3p and miR-27a-3p mimics simulate endogenous miR-23a-3p and miR-27a-3p. RCNs were transfected with either control vector (pmirGL0) or pmirGLO harboring both mi-R-23a-3p (pmiR-23aGLO) or miR-R27a-3p (pmiR-27aGL0) target sequences and cotransfected with miR mimics or hairpin inhibitors. Twenty-four hours after transfection, cells were analyzed for Luciferase activity. Normalized Luciferase activities were shown as the percentage relative to the control cells transfected with only reporter plasmid. $\boldsymbol{A}$, RCNs were cotransfected with pmirGLO or pmiR-23aGL0 or pmiR-27aGL0 and miR-R23a-3p, miR-R27a-3p, or negative control (-ve Con) mimics. $\boldsymbol{B}, \mathrm{RCNs}$ were cotransfected with pmirGL0 or pmiR-23aGLO or pmiR-27aGL0 and miR-R23a-3p, miR-R27a-3p, or negative control (-ve cnt) miR hairpin inhibitor. Data are mean \pm SD. ${ }^{* * *} p<0.001$ versus control cells $(N=3)$. RCNs were transfected with miR-R23a-3p, miR-R27a-3p, or negative control (-ve Con) mimics and treated with etoposide as described above. C, Analysis of qPCR data for miR-23a-3p and miR-27a-3p expression $24 \mathrm{~h}$ after etoposide treatment, normalized to U6 snRNA $(N=4)$. Data are expressed as percentage of control etoposide untreated neurons. Data are mean \pm SD. ${ }^{* *} p<0.001$ versus untreated neurons. ${ }^{+++} p<$ 0.01 versus negative control (-ve cnt) miR mimic transfected, etoposide-treated RCNs. Analysis by one-way ANOVA followed by multiple pairwise comparisons using Student-Newman-Keuls posthoc test. 
A

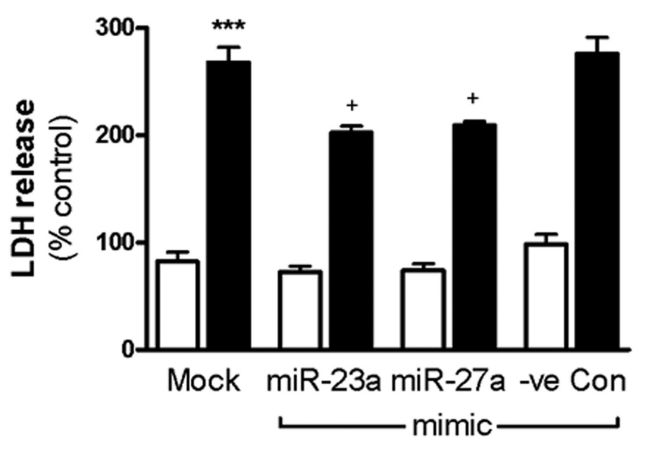

B

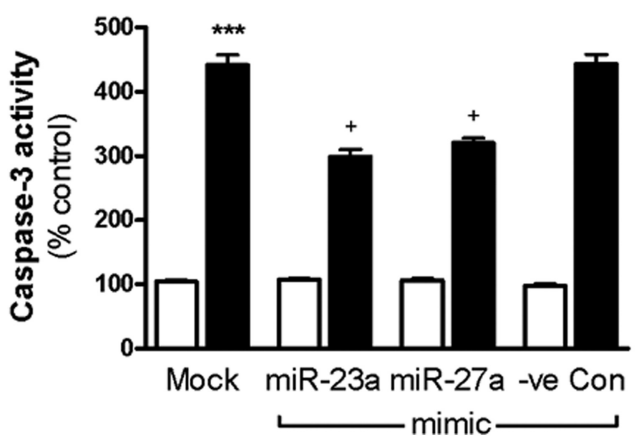

C miR hairpin inhibitors

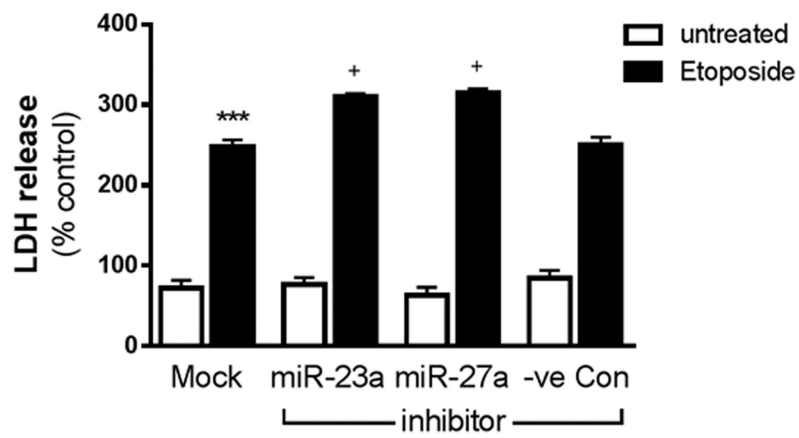

D

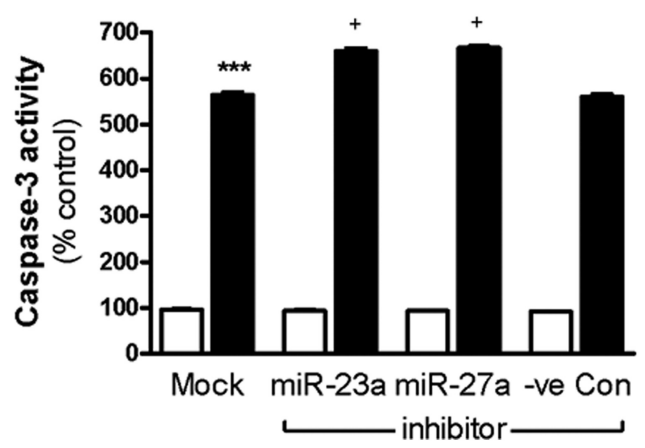

Figure 6. miR-23a and miR-27a mimics attenuate neuronal apoptosis and caspase-3 activation in etoposide-treated primary cortical neurons, whereas transfection with miR-23a and miR-27a hairpin inhibitors has opposite effects. Transfection of RCNs with miR-23a-3p and miR-27a-3p mimics significantly decreased etoposide-induced LDH release in in rat cortical neuronal cultures ( $\boldsymbol{A}$ ). Neurons were transfected with miR-23a-3p and miR-27a-3p mimics or negative control miR mimic (-ve Con mimic) and $4 \mathrm{~h}$ later treated with etoposide as described above. LDH release was measured after $24 \mathrm{~h}$ of treatment. Histograms indicate LDH release as percentage of control untreated RCNs. Transfection of RCNs with miR-23a-3p and miR-27a-3p 3p attenuates caspase-3 activation in etoposide-treated primary cortical neurons $(\boldsymbol{B})$. RCNs were transfected and etoposide treated as described above. Caspase-3-like activity was assayed fluorometrically by measuring the accumulation of free AMC resulting after cleavage of AC-DEVD-AMC. Data are expressed as percentage of control untreated neurons. Transfection of RCNs with miR-23a-3p and miR-27a-3p hairpin inhibitors increased etoposide-induced LDH release in in rat cortical neuronal cultures ( $($ ). Transfection of RCNs with miR-23a-3p and miR-27a-3p 3p hairpin inhibitors enhanced caspase-3 activation in etoposide-treated primary cortical neurons (D). RCNs were transfected and etoposide treated as described above. Caspase-3-like activity was assayed fluorometrically by measuring the accumulation of free AMC resulting after cleavage of AC-DEVD-AMC. Data are expressed as percentage of control etoposide-treated neurons. Data are mean $\pm S D$. ${ }^{*} p<0.05$ versus mock untreated RCNs. ${ }^{* *} p<0.01$ versus mock untreated RCNs. ${ }^{* * *} p<0.001$ versus mock untreated RCNs. ${ }^{+} p<0.05$ versus etoposide-treated mock $(N=4) .{ }^{++} p<0.01$ versus etoposide-treated mock $(N=$ 4). $N=8$ cultures per condition. Analysis by one-way ANOVA followed by multiple pairwise comparisons using Student-Newman-Keuls post hoc test.

totic members of Bcl-2 family, such as Puma, Noxa, Bax (Fig. $4 A$ ), and Bim (data not shown) after etoposide treatment compared with control samples. The expression of Puma showed the earliest increase $(1 \mathrm{~h})$, reached a peak at $6 \mathrm{~h}$, and declined at later time points. The expression of Noxa also increased rapidly and continued on an upward slope until 16-24 h. In contrast, gene expression of Bim (data not shown) and Bax showed significant increases only at 16-24 h. Quantitative Western blot analysis demonstrated that the protein levels of Puma, Noxa, Bax (Fig. 4B), and Bim (data not shown) were increased with a temporal profile similar, albeit not always identical, to the gene expression. Furthermore, the protein level of active Bax (Fig. 4B), as well as Bak (data not shown), was also increased by etoposide treatment.

Similar to TBI data, we did not observe any significant changes in XIAP expression after etoposide treatment (data not shown). We also observed downregulation of MAP4K4, MAP2K7, calpain-6, and calpain-7 after etoposide treatment compared with control samples (data not shown).
miR-23a-3p and miR-27a-3p mimics compensate for the decrease of endogenous miR-23a-3p, miR-27a-3p in etoposide-treated primary cortical neurons

To validate the ability of miR mimics to specifically replicate the function of endogenous miRs, RCNs were cotransfected with either control vector (pmirGLO) or pmirGLO harboring either mi-R23a-3p (pmiR-23aGLO) or mi-R27a-3p (pmiR-27aGLO) target sequences and miR-R23a-3p, miR-R27a-3p, or negative control mimics. Twenty-four hours after transfection, neurons were analyzed for Luciferase activity. As predicted, none of miR mimics affected Luciferase activity in neurons transfected with control vector (Fig. 5A). Relative Luciferase activity in neurons cotransfected with the pmiR-23aGlo and miR-23a mimic was 3 times lower compared with cells transfected with pmiR-23aGlo only. Neither miR-27a-3p nor negative control microRNA mimics affected Luciferase activity in neurons transfected with pmiR$23 \mathrm{aGLO}$ (Fig. 5A). Relative Luciferase activity in neurons cotransfected with the miR-27a-3p mimic and pmiR-27aGLO was 2.35 times lower compared with cells transfected with just 
A

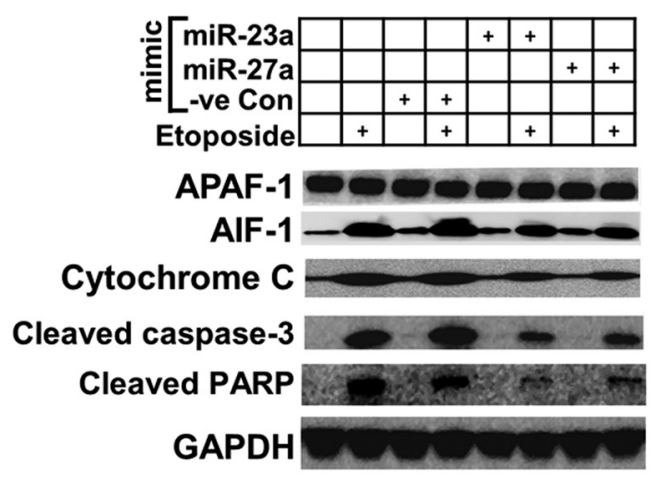

GAPDH
B

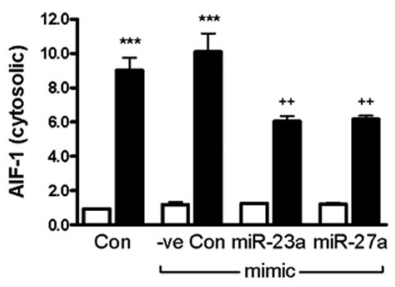

D

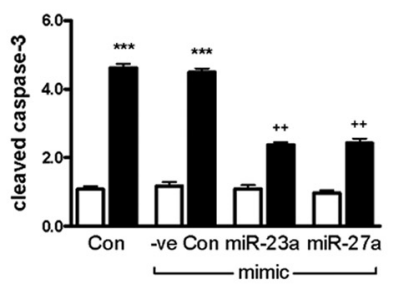

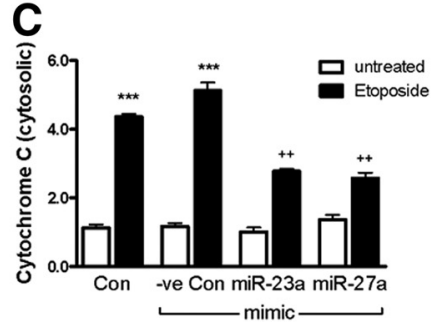

$\mathbf{E}$

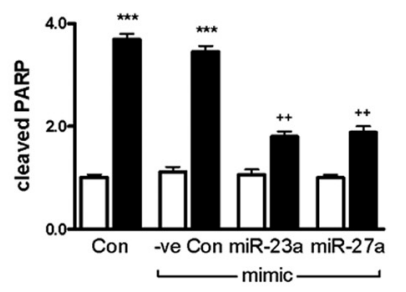

$\mathbf{F}$

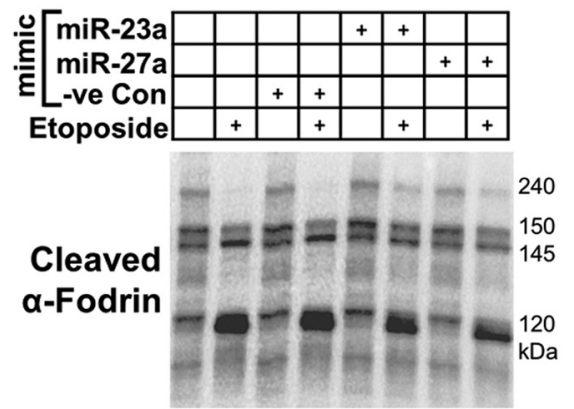

$\beta$-actin

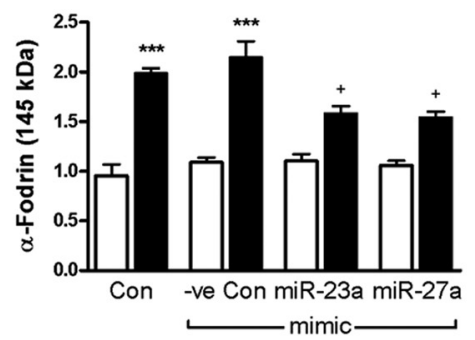

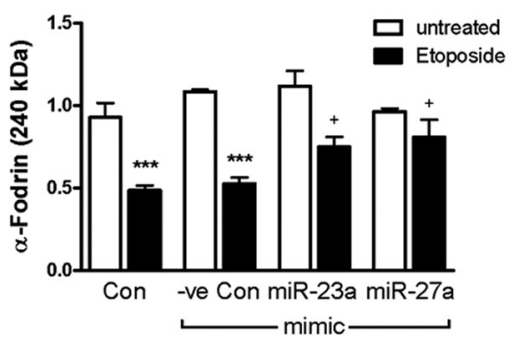

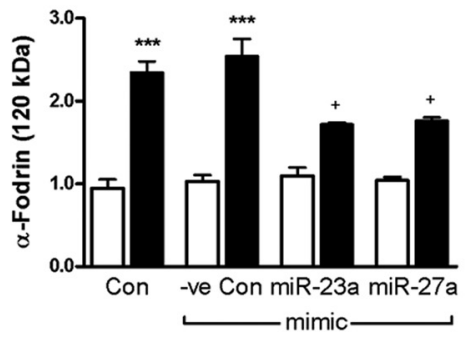

Figure 7. miR-23a and miR-27a mimics attenuate etoposide-induced release of AIF and cytochrome c into the cytosol as well as cleavage (activation) of caspase-3, PARP, and $\alpha$-fodrin. Transfection of RCNs with miR-23a and miR-27a decreases the levels of AIF-1, cytochrome $c$, and cleaved of caspase-3 and PARP in cytosolic fraction of etoposide-treated primary cortical neurons. Neurons were transfected with miR-23a-3p and miR-27a-3p mimics and $4 \mathrm{~h}$ later treated with etoposide as described above. Cytosolic fractions were fractioned on SDS-polyacrylamide gel and immunoblotted with antibodies against AIF-1, cytochrome $c$, cleaved caspase-3, PARP, GAPDH, and $\beta$-actin $(\boldsymbol{A})$. Levels of AIF-1(B), cytochrome c (C), cleaved caspase-3 (D), and PARP (E) proteins in cytosolic fraction of RCNs were quantified as fold change to levels of control etoposide-treated cells after measurement of band intensity by densitometry. Data are mean $\pm S D$. ${ }^{*} p<0.05$ versus mock untreated RCNs. ${ }^{* *} p<0.01$ versus mock untreated $R C N$ s. ${ }^{* * *} p<0.001$ versus mock untreated $R C N$ s. ${ }^{++} p<0.01$ versus etoposide-treated mock $(n=4)$. Whole-cell lysates were fractioned on SDS-polyacrylamide gel and immunoblotted with antibodies against $\alpha$-fodrin and $\beta$-actin $(\boldsymbol{F})$. Levels of $\alpha$-fodrin and products of its cleavage were quantified as fold change to control RCN level after measurement of band intensity by densitometry and normalization to levels of $\beta$-actin $(\boldsymbol{F})$. Data are mean \pm SD. ${ }^{*} p<0.05$ versus mock untreated RCNs. ${ }^{+} p<0.05$ versus etoposide-treated mock $(n=4)$. Analysis by one-way ANOVA followed by multiple pairwise comparisons using Student-Newman-Keuls post hoc test.

pmiR-27aGlo. miR-23a-3p and negative control microRNA mimics did not affect Luciferase activity in neurons transfected with pmiR-27aGLO (Fig. 5A). microRNA hairpin inhibitors are single-stranded RNA oligonucleotides designed to bind to and sequester the complimentary mature microRNA strand and prevent binding of miRs to their mRNAs targets, thereby blocking their activity. To validate the activity and specificity of microRNA hairpin inhibitors, RCNs were cotransfected with control vectorpmirGLO, pmiR-23aGLO, or pmiR-27aGLO and miR-R23a-3p, miR-R27a-3p, or negative control microRNA hairpin inhibitors. None of miR hairpin inhibitors affected Luciferase activity in neurons transfected with control vector -pmirGLO (Fig. 5B). Relative Luciferase activity in neurons cotransfected with the pmiR-23aGlo and miR-23a hairpin inhibitor was 2 times higher compared with cells transfected with just pmiR-23aGlo. Neither miR-27a-3p nor negative control microRNA mimics affected Luciferase activity in neurons transfected with pmiR-23aGLO (Fig. $5 B)$. Luciferase activity 2 times higher in neurons cotransfected with miR-27a-3p hairpin inhibitor and pmiR-27aGLO compared with cells transfected with pmiR-27aGlo only. miR-23a-3p and negative control microRNA mimics did not affect Luciferase activity in neurons transfected with pmiR-27aGLO (Fig. 5B). 
A

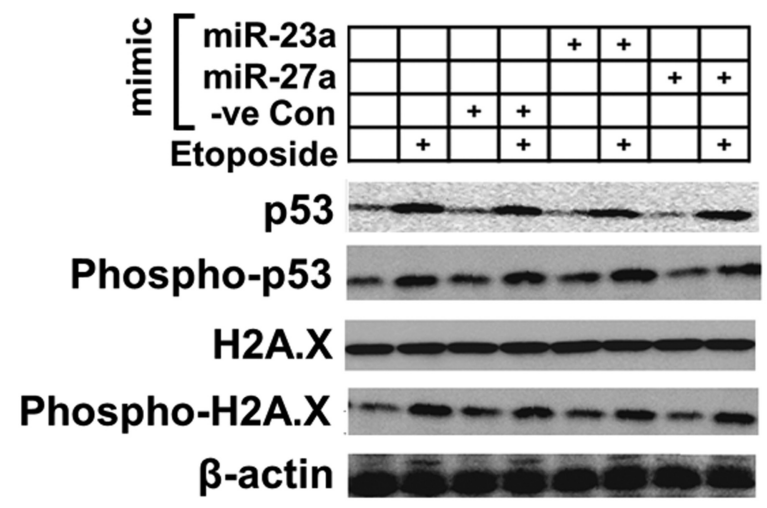

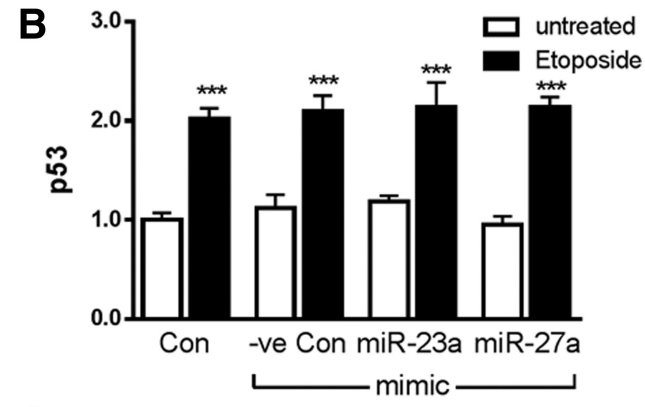

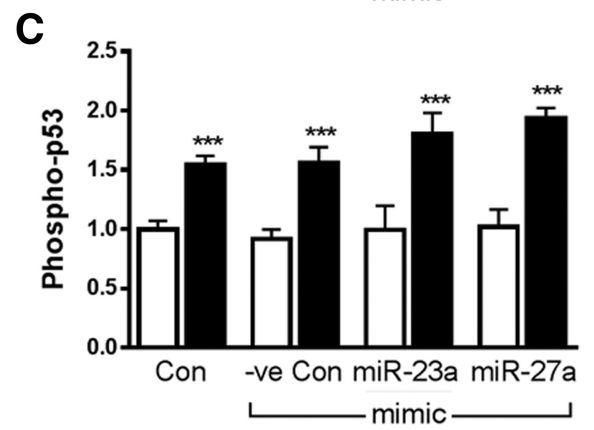

D

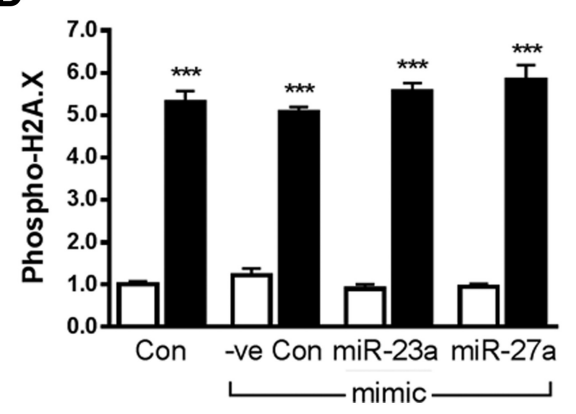

Figure 8. miR-23a and miR-27 do not alter etoposide-induced p53 activation or histone H2A.X phosphorylation. Neurons were transfected with miR-23a-3p and miR-27a-3p mimics and $4 \mathrm{~h}$ later treated with etoposide as described above. Whole-cell lysates were fractioned on SDS-polyacrylamide gel and immunoblotted with antibodies against p53, phosphorylated p53 (Ser 15), histone H2A.X, phosphorylated histone H2A.X (Ser 139), and $\beta$-actin (A). Levels of p53 (B), phosphorylated p53 (C), and phosphorylated histone H2A.X (D) were quantified as fold change to control RCN level after measurement of band intensity by densitometry and normalization to levels of $\beta$-actin. Data are mean $\pm S D .{ }^{*} p<0.05$ versus control untreated RCNs. ${ }^{* *} p<0.01$ versus control untreated RCNs. ${ }^{* * *} p<0.001$ versus control untreated RCNs. ${ }^{+} p<0.05$ versus etoposide-treated mock $(n=4) .{ }^{++} p<0.01$ versus etoposide-treated mock $(n=4) .{ }^{+++} p<0.001$ versus etoposide-treated mock $(n=4)$. Analysis by one-way ANOVA followed by multiple pairwise comparisons using Student-Newman-Keuls post hoc test.

To test the hypothesis that miR-23a-3p and miR-27a-3p decline plays a significant role in etoposide-induced neuronal apoptosis, we transfected primary cortical neurons with miR-23a-3p and miR-27a-3p mimics before etoposide treatment. These mimics are intended to compensate for the decline in endogenous miR-23a-3p and miR-27a-3p levels. The level of miR-23a in control neurons transfected with the miR-23a mimic was 5.7 times increased compared with untransfected control cells; the level of miR-23a in etoposide-treated neurons transfected with the miR-23a mimic was 4.25 times increased compared with untransfected control cells, more than compensating for the etoposide-induced decline in endogenous miR (Fig. 5C). The level of miR-27a in neurons transfected with the miR-27a mimic was 2.5 times increased compared with untransfected control cells; the level of miR-27a in etoposide-treated neurons transfected with the miR-27a mimic was 1.69 times higher compared with untransfected control cells, more than compensating for the etoposide-induced decline in endogenous miR (Fig. 5C). Importantly, attenuation of miR23a and miR-27a decline by transfection with the miR-24-3p or miR-27a-3p mimics significantly reduced etoposide-induced neuronal cell death (LDH assay) compared with mock transfection and miR mimic-negative controls (Fig. 6A). Similarly, etoposide-induced caspase-3 activity was significantly reduced in neurons transfected with miR-24-3p or miR-27a-3p mimics compared with mock transfection and microRNA mimic-negative controls (Fig. 6B). These data indicate that miR-23a and miR-27a are negative regulators of neuronal apoptosis.

We also used the miR hairpin inhibitor approach to confirm that decreased miR-24-3p and miR-27a-3p functional activity promotes neuronal apoptosis and caspase-3 activation after etoposide treatment. RCN cultures were transfected with miR-24-3p and miR-27a-3p hairpin inhibitors followed by treatment with etoposide. Consistent with our hypothesis, we observed a significant increase in etoposide-induced neuronal cell death (LDH assay) and caspase-3 activity in cells transfected with miR-24-3p and miR-27a-3p hairpin inhibitors compared with mock transfection and microRNA hairpin inhibitor-negative controls (Fig. $6 C, D)$. Transfection of neurons with miR-24-3p and miR-27a-3p hairpin inhibitors did not cause a significant induction of apoptosis in the absence of etoposide, suggesting that changes in 


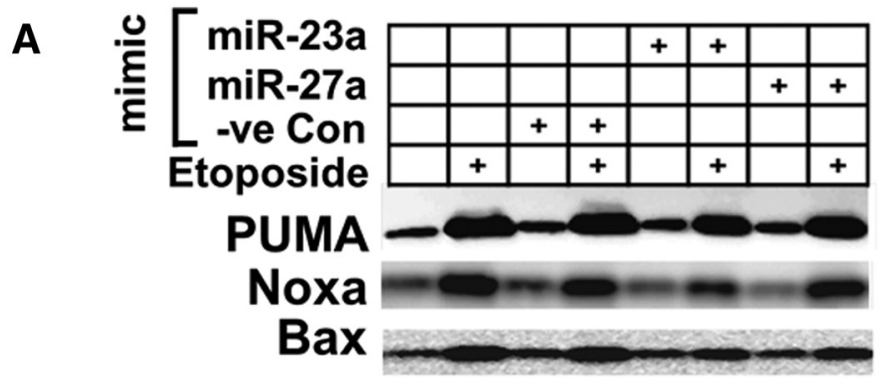

Active Bax

$\beta$-actin

B

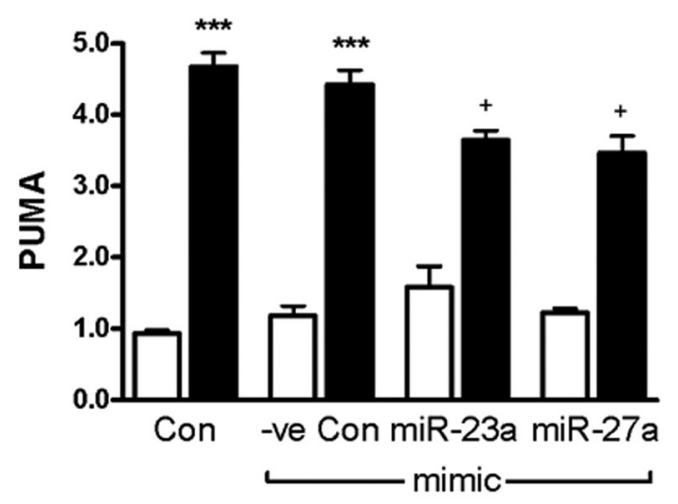

D

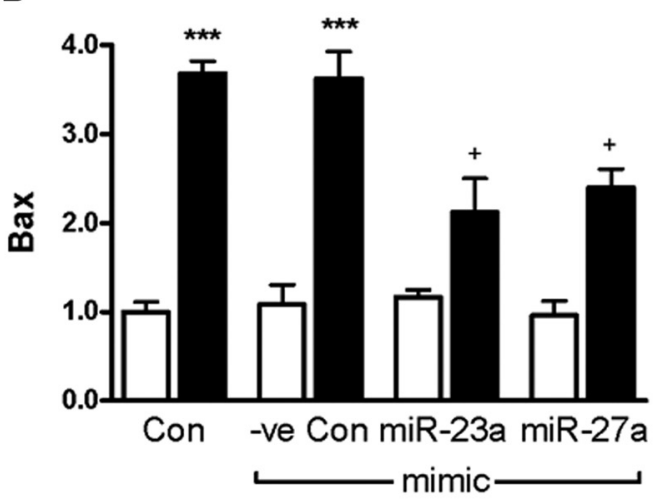

C

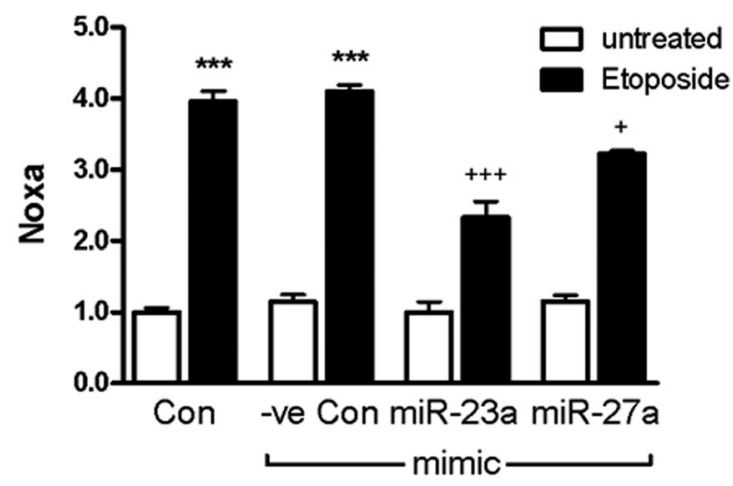

E

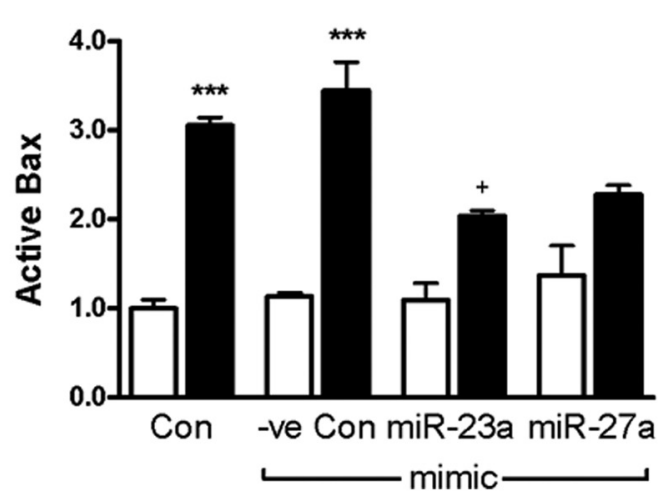

Figure 9. miR-23a and miR-27a mimics attenuate etoposide-induced expression of proapoptotic Bcl-2 family members. Neurons were transfected with miR-23a-3p and miR-27a-3p mimics and $4 \mathrm{~h}$ later treated with etoposide as described above. Whole-cell lysates were fractioned on SDS-polyacrylamide gel and immunoblotted with antibodies against PUMA, Noxa, Bax, active Bax, and $\beta$-actin $(\boldsymbol{A})$. Levels of PUMA $(\boldsymbol{B})$, Noxa $(\boldsymbol{C})$, Bax $(\boldsymbol{D})$, and active Bax $(\boldsymbol{E})$ were quantified as fold change to control RCN level after measurement of band intensity by densitometry and normalization to levels of $\beta$-actin. Data are mean \pm SD. ${ }^{*} p<0.05$ versus mock untreated RCNs. ${ }^{* *} p<0.01$ versus mock untreated RCNs. ${ }^{* * *} p<0.001$ versus mock untreated RCNs. ${ }^{+} p<0.05$ versus etoposide-treated mock $(n=4) .{ }^{++} p<0.01$ versus etoposide-treated mock $(n=4) .{ }^{+++} p<0.001$ versus etoposide-treated mock $(n=4)$. Analysis by one-way ANOVA followed by multiple pairwise comparisons using Student-Newman-Keuls post hoc test.

these miRs are not sufficient on their own to cause neuronal cell death.

miR-23a and miR-27a mimics attenuate molecular mechanisms of neuronal apoptosis

One of the most important steps in apoptosis involves the release of cytochrome $c$ and AIF from the mitochondria to initiate the intrinsic caspase activation pathway and a caspase-independent cell death pathway, respectively. To assess whether changes in
miR-24-3p and miR-27a-3p act upstream of these events, we examined cells transfected with miR mimics. Quantitative Western blot analysis demonstrated that miR-24-3p and miR-27a-3p mimics, with or without etoposide treatment, did not show changes of protein levels for key proapoptotic proteins, including apoptotic peptidase activating factor 1 (APAF1), apoptosisinducing factor, mitochondrion-associated 1 (AIF-1), cytochrome $c$, and TNF receptor superfamily member 6 (Fas) in whole-cell lysates (data not shown). Translocation of AIF and 


\section{A PUMA-GLO}

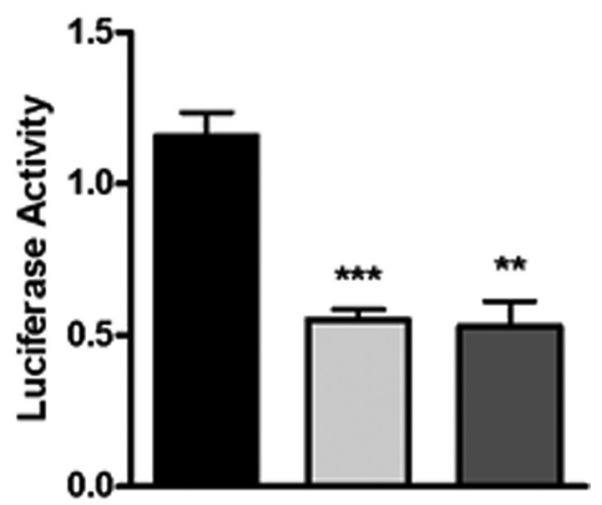

\section{B Noxa-GLO}

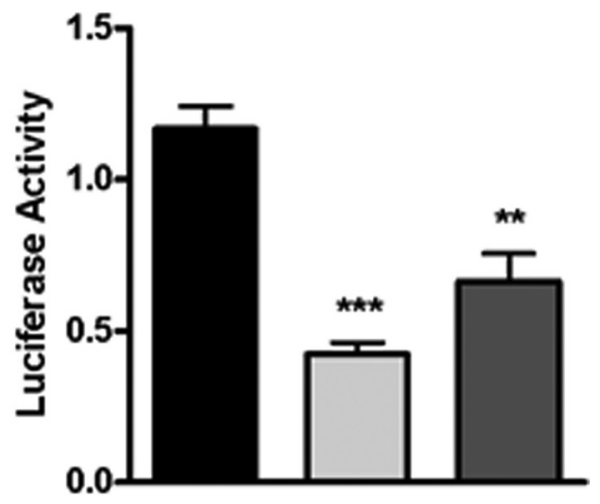

\section{Bax-GLO}

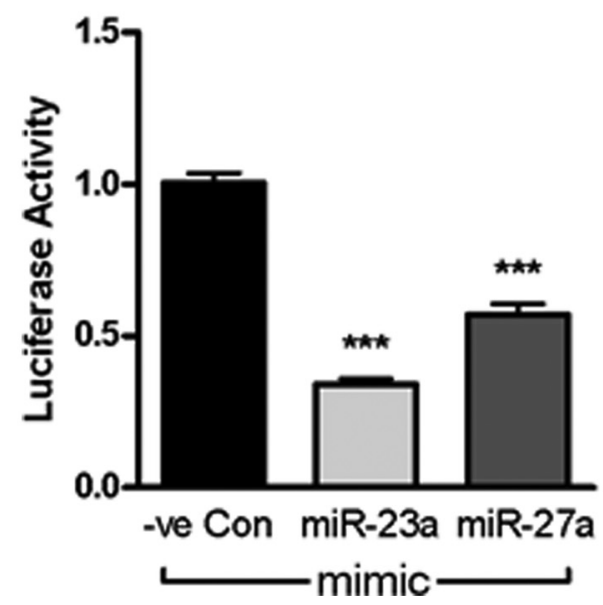

Figure 10. miR-23a and miR-27a target PUMA, Noxa, and Bax. SH-SY5Y cells were transfected with either negative control miR (-ve cnt) or miR-23a-3p or miR-27a-3p mimics. Cells were also cotransfected with reporter plasmids with inserted 3' UTRs of mouse PUMA (A), Noxa (B), and Bax (C). Twenty-four hours after transfection, cells were analyzed for Luciferase activity. Normalized Luciferase activities were shown as the percentage relative to the cells transfected with reporter plasmid and -ve Con miR mimic, which was set as 1. Experiments were performed in triplicate. Data are mean \pm SD. ${ }^{* *} p<0.01(n=3) .{ }^{* *} p<0.001(n=3)$. Analysis by one-way ANOVA followed by multiple pairwise comparisons using Student-Newman-Keuls post hoc test. cytochrome $c$ from mitochondria to cytoplasm is a well-known marker of apoptotic cell death (Sabirzhanov et al., 2012). Analysis of cytosolic fractions revealed that miR-24-3p and miR-27a-3p mimics significantly reduced etoposide-induced release of AIF and cytochrome $c$ into the cytosol (Fig. 7A-C). In addition, miR24-3p and miR-27a-3p mimics significantly attenuated etoposide-induced cleavage of caspase-3 (Fig. $7 A, D$ ), as well as cleavage of PARP and $\alpha$-fodrin, well-known caspase substrates whose cleavage is a good indicator of caspase activity. Quantitative Western blot analysis demonstrated that neurons transfected with miR-24-3p and miR-27a-3p mimics displayed significantly reduced levels of the cleaved fragment of PARP (Fig. $7 A, E$ ) and significantly attenuated the decrease of the full-length uncleaved fragment of $\alpha$-fodrin ( $240 \mathrm{kDa}$ ) (Fig. $7 F$ ) after etoposide treatment. In addition, neurons transfected with the mimics had significantly reduced levels of the levels of both calpain-dependent $(150 / 145 \mathrm{kDa})$ and caspase-dependent cleavage $(150 / 120 \mathrm{kDa})$ (Siman et al., 1984, 2004; Cryns et al., 1996) fragments of $\alpha$-fodrin after etoposide treatment (Fig. $7 F$ ).

miR-23a and miR-27 mimics act downstream of p53 activation

To explore at a molecular level the effect of miR-23a and miR-27a on DNA damage-induced p53-dependent cell death pathways, we analyzed the effect of miR-23a and miR-27a mimics on pathways downstream of p53 activation. Transfection of RCNs by miR-24-3p and miR-27a-3p mimics did not attenuate etoposide-induced increases in phosphorylated histone H2A.X (Ser 139) or p53 protein levels expression and/or phosphorylation (Fig. 8).

miR-23a and miR-27a mimics attenuate expression of proapoptotic Bcl-2 family members during apoptosis We also investigated the protein levels of key proapoptotic members of Bcl-2 family in neurons transfected with miR-23-3p and miR-27a-3p mimics followed by etoposide treatment. Quantitative Western blot analysis demonstrated that the mimics significantly attenuated the etoposide-induced increase in PUMA, Noxa, Bax, and active Bax levels (Fig. 9) but not Bim or BAK1 (data not shown) expression levels.

\section{miR-23a and miR-27a target $3^{\prime}$ UTRs of PUMA, Noxa,} and Bax

miRNA target prediction tools from mIRecords were used to predict mRNA targets for miR-23a and miR-27a (seed parameter settings: minimum seed size 7; allow single G:U; allow single mismatch). Sixteen sites for miR-23a-3p and 14 sites for miR-27a-3p were predicted in mouse Noxa 3' UTR (NCBI Reference Sequence: NM_021451.2); 4 sites for miR-23a-3p and 7 sites for miR-27a-3p were predicted in mouse PUMA 3'UTR (NCBI Reference Sequence: BC044782.2); 1 site for miR23a-3p and 4 sites for miR-27a-3p were predicted in mouse Bax 3'UTR (NCBI Reference Sequence: BC053380.1). To directly test whether miR-23a-3p and miR-27a-3p could functionally target PUMA, Noxa, and Bax mRNAs, we used a Luciferase reporter assay in which the 3' UTRs of mouse PUMA, Noxa, and Bax, were inserted $3^{\prime}$ of the firefly Luciferase gene into pmirGLO plasmid. Each Luciferase construct was cotransfected with either negative control, miR23a-3p, or miR-27a-3p microRNA mimics into SH-SY5Y cells, and Luciferase activity was measured $24 \mathrm{~h}$ after transfection. miR-23a-3p and miR-27a-3p, but not negative control miR mimic, significantly reduced Luciferase activity in cells trans- 
A

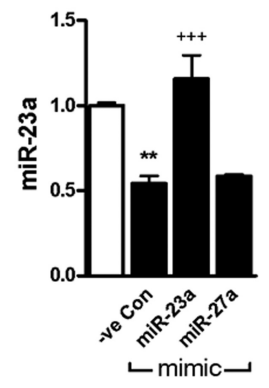

$\mathbf{B}$

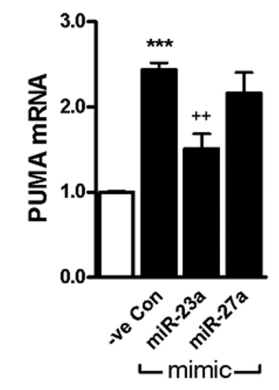

C

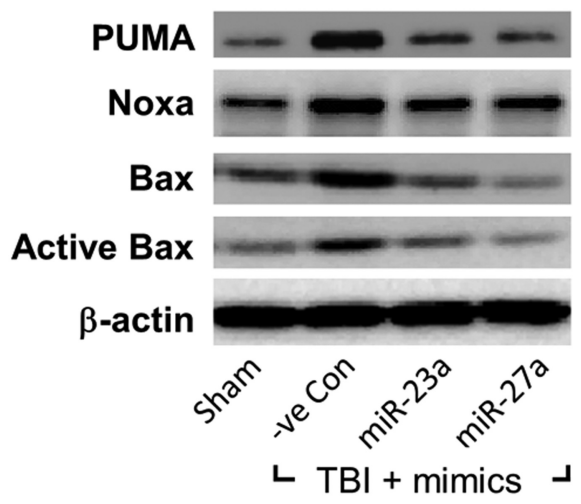

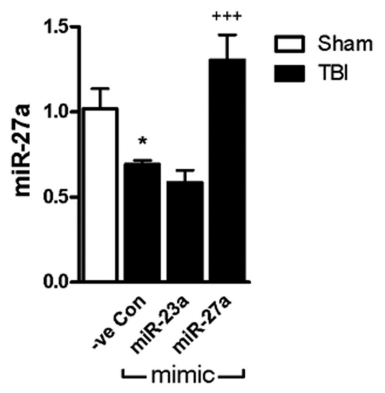
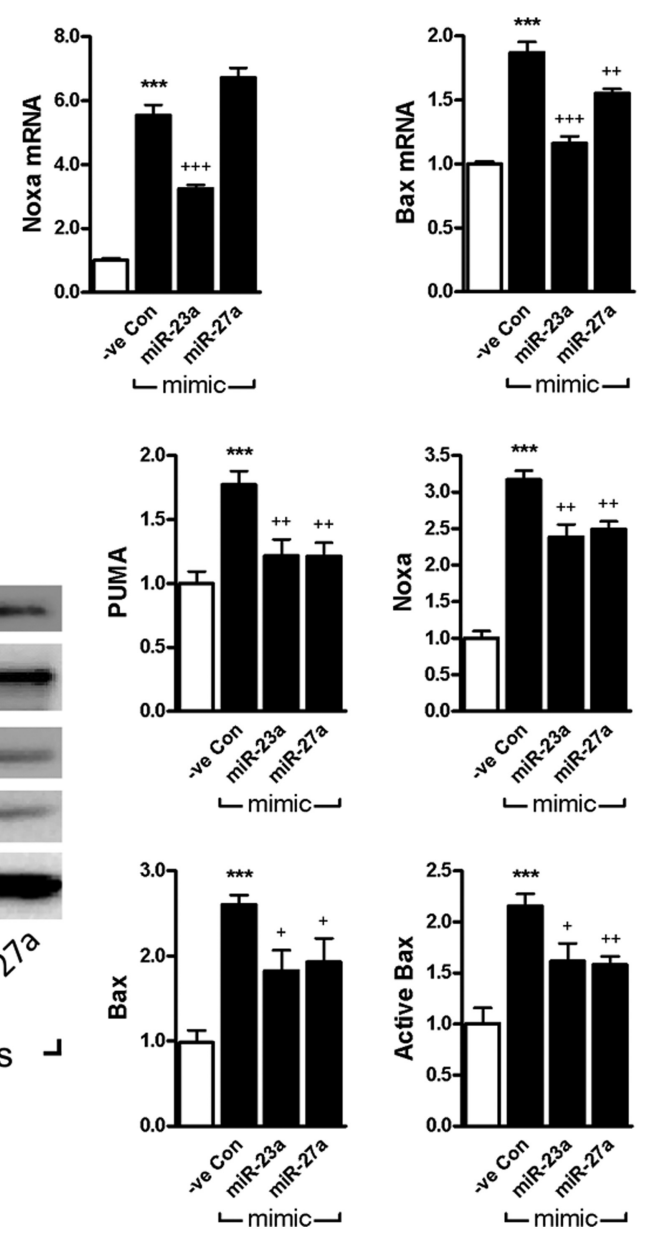

Figure 11. Intracerebroventricular injection of miR-23a and miR-27a mimics attenuate expression of PUMA, Noxa, and Bax in injured cortex after TBI. qPCR quantification of miR-23a-3p, miR-27a-3p ( $\boldsymbol{A}$ ) and PUMA, Noxa, and Bax (B) expressions in mouse cortex $6 \mathrm{~h}$ after TBl and intracerebroventricular injection of miR-23a-3p or miR-27a-3p or negative control miR (-ve Con) mimics. Levels of miRs were normalized to U6 snRNA; levels of PUMA, Noxa, and Bax were normalized to GAPDH. Data are mean \pm SD. ${ }^{*} p<0.05$ versus sham animals. ${ }^{* *} p<0.01$ versus sham animals. ${ }^{* *} p<0.001$ versus sham animals. ${ }^{+} p<0.05$ versus injured -ve Con injected group $(N=4-6) .{ }^{++} p<0.01$ versus injured -ve Con injected group $(N=4-6)$. C, Whole-tissue lysates from mouse cortex $24 \mathrm{~h}$ after TBI and intracerebroventricular injection of miR-23a-3p or miR-27a-3p or -ve cnt mimics were fractioned on SDS-polyacrylamide gel and immunoblotted with antibodies against PUMA, Noxa, Bax, active Bax, and $\beta$-actin. Levels of PUMA, Noxa, Bax, and active Bax in total lysates were quantified as fold change to the levels of sham after measurement of band intensity by densitometry. Data are mean $\pm S D .{ }^{*} p<0.05$ versus sham animals. ${ }^{* *} p<0.01$ versus sham animals. ${ }^{* *} p<$ 0.001 versus sham animals. ${ }^{+} p<0.05$ versus injured -ve cnt-injected group $(N=4-6) .{ }^{++} p<0.01$ versus injured -ve cnt-injected group $(N=4-6)$.

fected with constructs containing 3' UTRs of PUMA, Noxa, and Bax (Fig. 10). Together, these data demonstrate that miR$23 a-3 p$ or miR-27a-3p is capable of targeting sequences in the 3' UTRs of PUMA, Noxa, and Bax mRNAs and inhibit expression of mRNA that include these sequences.

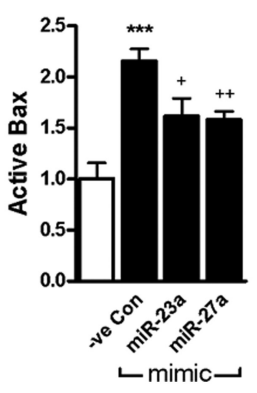

Intracerebroventricular injection of miR-23a and miR-27a mimics attenuate expression of PUMA, Noxa, and Bax and markers of apoptosis after TBI We administered miR-23a-3p and miR27a-3p mimics after TBI and examined the expression of proapoptotic $\mathrm{Bcl}-2 \mathrm{fam}$ ily members PUMA, Noxa, and Bax. At 15 min after injury, mice received a single intracerebroventricular injection of either miR-23a, miR-27a, or negative control miR mimics. Levels of miR-23a-3p, miR27a-3p, Puma, Noxa, and Bax mRNA were analyzed by qPCR in the injured cortex $6 \mathrm{~h}$ after TBI. Our data demonstrate that injection of miR-23a and miR-27a mimics completely reversed the TBIinduced decline in miR-23a and miR-27a, respectively, compared with TBI mice injected with negative control $\mathrm{miR}$ mimic (Fig. 11A). Importantly, injection of miR$23 a-3 p$ and miR-27a-3p mimics significantly attenuated TBI-induced upregulation of Puma, Noxa, and Bax mRNA compared with TBI mice injected with negative control miR mimic (Fig. 11B). In addition, miR-23a-3p and miR-27a-3p mimics also significantly attenuated the TBI-induced increase in PUMA, Noxa, Bax, and active Bax protein levels in TBI cortex at $24 \mathrm{~h}$ after injury compared with negative control miR mimics (Fig. 11C).

We also examined the effect of intracerebroventricular injection with miR-23a$3 \mathrm{p}, \operatorname{miR}-27 \mathrm{a}-3 \mathrm{p}$, and negative control miR mimics on multiple apoptosis markers in the TBI cortex at $24 \mathrm{~h}$ after injury. Quantitative Western blot analysis demonstrated that miR-23a-3p and miR$27 a-3 p$ did not change the levels of key proapoptotic proteins, such as AIF-1 and cytochrome $c$, in whole-tissue lysates compared with negative control miR mimics. In addition, intracerebroventricular injection of miR-23a-3p and miR$27 a-3 p$ mimics significantly attenuated cleavage of $\alpha$-fodrin (Fig. 12A). Analysis of cytosolic extracts revealed that miR23-3p and miR-27a-3p mimic significantly reduced TBI-induced release of AIF and cytochrome $c$ into the cytosol (Fig. 12B).

miR-23a mimic treatment reduced lesion volume and neuronal loss in the hippocampus after TBI

TBI-induced lesion volume at $28 \mathrm{~d}$ after injury was quantified using stereological methods in cresyl violet-stained coronal brain sections from TBI mice that received either negative control miR mimic or miR-23a-3p mimic by intracerebroventricular injection (chosen based on the relative strength of antiapoptotic effects). miR-23a-3p injection significantly reduced TBI-induced lesion volumes compared with the negative control miR mimic 

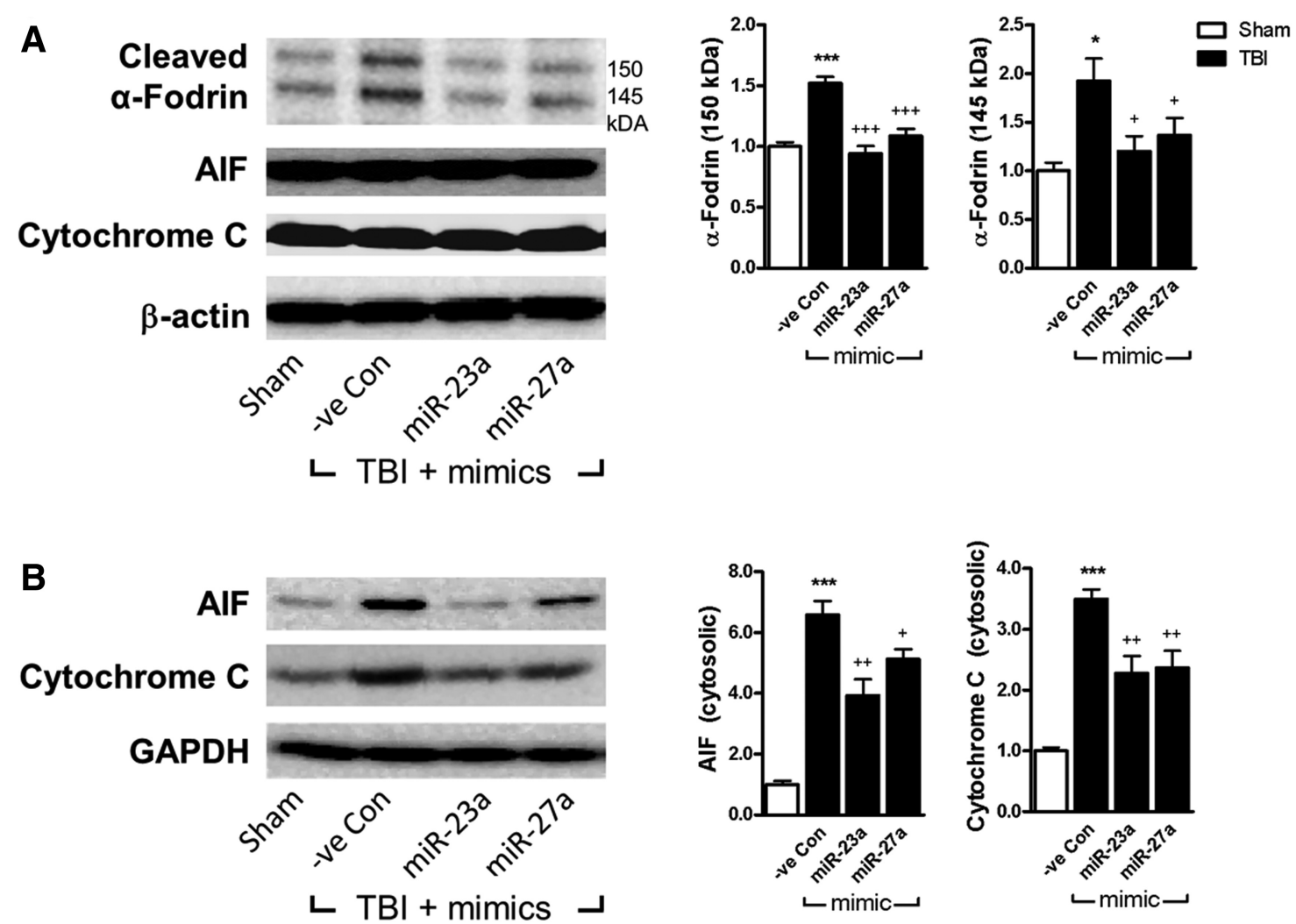

Figure 12. Intracerebroventricular injection of miR-23a and miR-27a mimics attenuate neuronal apoptosis in injured cortex after TBI. $A$, Whole-tissue lysates from mouse cortex $24 \mathrm{~h}$ after TBI and intracerebroventricular injection of miR-23a-3p or miR-27a-3p or -ve Con mimics were fractioned on SDS-polyacrylamide gel and immunoblotted with antibodies against $\alpha$-fodrin, AlF-1, cytochrome $c$, and $\beta$-actin. Levels of cleaved $\alpha$-fodrin, AlF-1, and cytochrome $c$ in total lysates were quantified as fold change to the levels of sham after measurement of band intensity by densitometry. Data are mean $\pm S D .{ }^{*} p<0.05$ versus sham animals. ${ }^{* *} p<0.01$ versus sham animals. ${ }^{* * *} p<0.001$ versus sham animals. ${ }^{+} p<0.05$ versus injured - ve Con miR mimics injected group $(N=4-6) .{ }^{++} p<0.01$ versus injured -ve Con miR mimics injected group $(N=4-6) .{ }^{+++} p<0.001$ versus injured -ve Con miR mimics injected group $(N=4-6) . B$, Cytosolic fractions from cortex tissues $24 \mathrm{~h}$ after TBI and intracerebroventricular injection of miR-23a-3p or miR-27a-3p or -ve cnt mimics were fractioned on SDS-polyacrylamide gel and immunoblotted with antibodies against AIF-1, cytochrome $c$, and GAPDH. Levels of AlF-1 and cytochrome c proteins in cytosolic fraction of TBI miR mimics injected animals were quantified as fold change to the levels of sham after measurement of band intensity by densitometry. Data are mean $\pm S D .{ }^{*} p<0.05$ versus sham animals. ${ }^{* *} p<0.01$ versus sham animals. ${ }^{* * *} p<0.001$ versus sham animals. ${ }^{+} p<0.001$ ve 0.05 versus injured -ve cnt miR mimics injected group $(N=4-6) .{ }^{++} p<0.01$ versus injured -ve cnt miR mimics injected group $(N=4-6) .{ }^{++} p<0.001$ versus injured -ve cnt miR mimics injected group $(N=4-6)$. Analysis by one-way ANOVA followed by multiple pairwise comparisons using Student-Newman-Keuls post hoc test.

group (Fig. $13 \mathrm{~A}, \mathrm{~B}$; -ve control $=4.561 \pm 0.4099 \mathrm{~mm}^{3}$ and $\mathrm{miR}$ $\left.23 \mathrm{a}=2.941 \pm 0.6468 \mathrm{~mm}^{3}, n=9\right)$. TBI-induced neuronal loss in the CA1, CA2/3, and dentate gyrus subregions of hippocampus at $28 \mathrm{~d}$ after injury was also quantified by stereological methods from sham-injured, TBI + negative control miR mimic, and TBI + miR-23a-3p mimic groups. No significant effect of miR-23a mimic treatment was observed in CA1 neuronal densities (Fig. $13 C)$, although a neuroprotective trend was observed. Importantly, administration of miR-23a-3p significantly attenuated TBI-induced neuronal loss in CA2/3 (Fig. 13D) and dentate gyrus (Fig. 13E) compared with the TBI-negative control miR mimic group.

\section{Discussion}

The modulation of apoptotic mechanisms by select miRs has been described in cerebral ischemia models (Siegel et al., 2011; Selvamani et al., 2012). miR-29b inhibits apoptosis during neuronal maturation by targeting proapoptotic $\mathrm{BH} 3$-only genes (Kole et al., 2011), and miR-223 is neuroprotective by lowering levels of specific glutamate receptors (Harraz et al., 2012). Recent in vitro studies demonstrated that miR-34a, miR-451, and miR874 increased vulnerability to injury in transfected neurons (Truettner et al., 2013). In a rat TBI model, Redell et al. (2011) described changes impacting miR-21 and some of its known and predicted targets, but without demonstrating a causal relationship between miRNA changes and TBI-induced neuronal cell death. We present the first detailed characterization of specific miR changes in the injured cortex after TBI, and the elucidation of their effects on neuronal cell death mechanisms. We performed a temporal profiling of miRNAs that were altered in the acute period after experimental TBI in mice, and selected miRs, which were rapidly downregulated in the first $24 \mathrm{~h}$ after TBI, a time period associated with intense activation of neuronal cell death pathways (Stoica and Faden, 2010). Of 36 miRs downregulated after TBI (data not shown), only three (miR-23a-3p, miR$27 a-3 p$, and miR-143) declined rapidly in the first hours after trauma and returned to normal levels several days later. We hypothesized that this pattern reflects an active regulation and selective involvement in the acute secondary neuronal cell death mechanisms that characterize the early post-traumatic period (Di Giovanni et al., 2003). We concentrated on miR-23a and miR$27 \mathrm{a}$ because they are the members of the same genomic cluster.

Changes in miR-23a and miR-27a after TBI in rat have been reported, but no detailed analysis (qPCR) or mechanistic evaluation was performed. Hu et al. (2012) showed that miR-23a* (miR-23a-5p), different from the miR-23a-3p under investigation here, was increased at $24 \mathrm{~h}$ after rat CCI in the injured hip- 
A

-ve Control
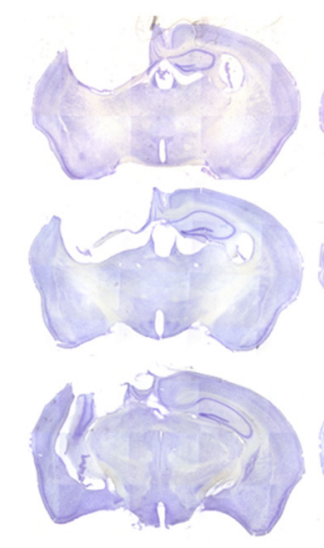

C

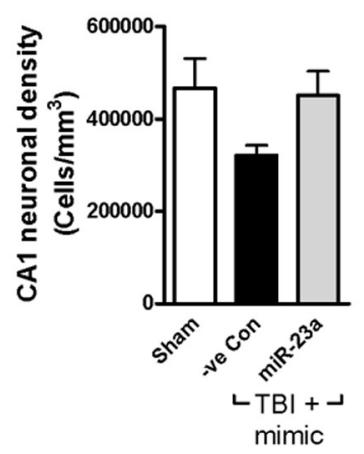

miR-23a
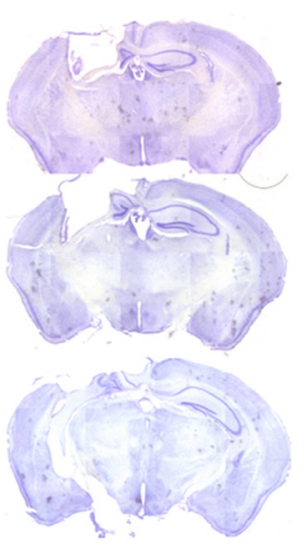

D

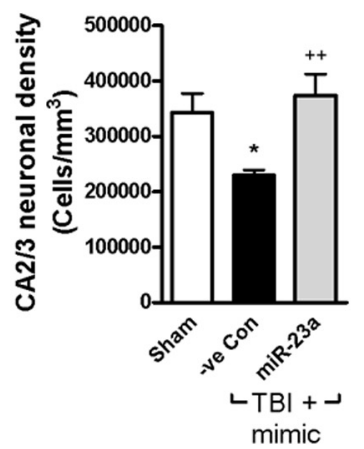

B

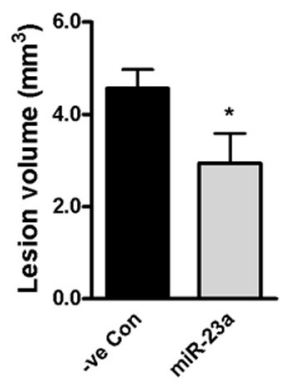

E

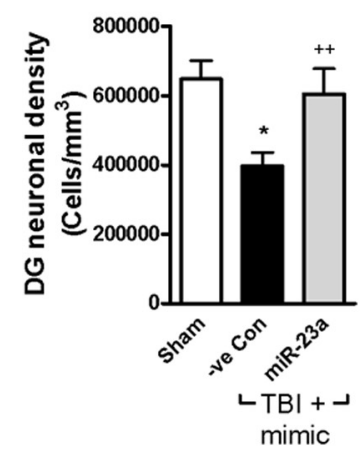

Figure 13. miR-23a treatment reduced lesion volume and neuronal loss in the cortex after TBI. Lesion volume was quantified using the Cavalieri method. Unbiased stereological assessment of lesion volume at $28 \mathrm{~d}$ after TBI was performed on cresyl violetstained brain section. $\boldsymbol{A}$, Representative images from each group are shown. $\boldsymbol{B}$, Significant reduction of lesion volume was observed in miR-23a-3p mimic-treated group. ${ }^{*} p<0.05$, compared with the TBI-negative miR mimic group (Student's $t$ test). Data are mean \pm SEM; $n=9$. Stereological assessment of neuronal cell on PID28 was performed on cresyl-violet stained sections in the CA1 $(\boldsymbol{C}), C A 2 / 3(\boldsymbol{D})$, and DG $(\boldsymbol{E})$ subregions of cortex. Significant differences of neuronal density were observed between sham-injured and TBI-negative miR mimic groups in the CA2/3 $\left({ }^{*} p<0.05\right)$ and DG $\left({ }^{*} p<0.05\right)$ subregions of cortex. miR-23a-3p mimic treatment significantly increased neuronal density in the CA2/3 $\left({ }^{\# \#} p<0.01\right)$ and DG ( $\left.{ }^{\# \#} p<0.01\right)$ cortex compared with TBInegative miR mimic group. Analysis by one-way ANOVA followed by Student-Newman-Keuls test. Data are mean \pm SEM; $n=$ $6-9$.

pocampus. Another study mentioned miR-23a-3p expression after rat lateral fluid percussion, but the data as presented were insufficient to determine whether changes were significant (Lei et al., 2009). Truettner et al. (2011) observed that miR-27a and miR-27b were decreased in the injured cortex at $7 \mathrm{~h}$ after rat TBI after treatment with hypothermia, although the data with normothermia were inconclusive. Another recent study reported that miR-27a increased in the hippocampus after status epilepticus, but the relationship to cell death was not examined (JimenezMateos et al., 2011).

We hypothesized that decreases of miR-23a-3p and miR$27 a-3 p$ may result in the upregulation of their proapoptotic targets. mRNA target prediction analysis (TargetScan, miRecords, PITA) identified multiple proapoptotic genes that are potential targets for miR-23a-3p and miR-27a-3p, including the p53 tumor suppressor protein, a key modulator of DNA damageinduced cell death. p53 activation is mediated via phosphorylation at Ser 15 and Ser20 (Shieh et al., 1997), and we observed rapid upregulation and activation/phosphorylation of p53 at Ser15 af- ter TBI. DNA damage in neurons results in increased levels of phosphorylated histone H2A.X (Ser139) (Rogakou et al., 1999) and induces p53 upregulation and activation/phosphorylation. In turn, p53 induces various proapoptotic Bcl2 family molecules and apoptosis (Lowe et al., 1993).

Other predicted targets for miR23a-3p and miR-27a-3p include proapoptotic members of Bcl-2 family, such as BH3-only members PUMA and Noxa as well as multi- $\mathrm{BH}$ domain $\mathrm{Bcl} 2$ family member BAX (Oda et al., 2000; Nakano and Vousden, 2001; Kaeser and Iggo, 2002). After TBI, we observed rapid increase of mRNA levels of PUMA and Noxa. Their expression peaked between 1 and $24 \mathrm{~h}$ after injury and later declined, consistent with regulation by miR-23a-3p and miR-27a-3p. Protein analysis confirmed that TBI induced rapid increases in the levels of Puma, Noxa, and Bax. PUMA and Noxa induce direct as well as indirect activation of Bax resulting in its translocation to the mitochondria followed by mitochondrial outer membrane permeabilization and the release of mitochondrial apoptogenic proteins, including cytochrome $c$ and AIF-1 (Liu et al., 1996; Susin et al., 1999; Nakano and Vousden, 2001). Notably, cortical neurons are resistant to apoptosis in the absence of PUMA and Noxa (Steckley et al., 2007).

miR-23a and miR-27a downregulation occurred rapidly after etoposide treatment and lasted for at least $24 \mathrm{~h}$; and as in the in vivo TBI model, it was paralleled by significant increases in PUMA, Noxa, and Bax (total and activated). The expression of miR-23-3p and miR-27a-3p in neuroglia was much smaller compared with primary neurons (data not shown), suggesting that the changes observed in vivo likely reflect neuronal events.

Etoposide induces caspase-dependent and caspaseindependent neuronal apoptosis via release from mitochondria into the cytosol of cytochrome $c$ and AIF, respectively (Culmsee and Mattson, 2005). The proapoptotic effect of these molecules in neuronal apoptosis involves changes in subcellular localization without modifying expression levels (Sabirzhanov et al., 2012). Cytosolic cytochrome $c$ leads to activation of caspases (Li et al., 1997; Srinivasula et al., 1998), cleavage of caspase substrates, such as PARP and cell death (Le Rhun et al., 1998). Cytosolic AIF-1 translocates to the nucleus mediating caspase-independent apoptosis (Susin et al., 1999). After both mouse CCI in vivo and etoposide treatment in primary cortical neurons in vitro, we observed cytochrome $c$ release and PARP cleavage as well as AIF release.

Administration of miR-23a and miR-27a mimics resulted in attenuation of etoposide-induced neuronal cell death (p53dependent apoptosis) and caspase activity. Conversely, treatment with miR-23a and miR-27a hairpin inhibitors, which attenuate 
miR levels, enhanced neuronal apoptosis. Together, these data demonstrate the important role played by miR-23a and miR-27a decline in neuronal cell death. Neither miR-23a nor miR-27a mimics affected p53 expression or phosphorylation, or caused changes in histone H2A.X phosphorylation. We conclude that miR-23a and miR-27a modulate neither the etoposide-induced DNA damage nor the subsequent activation of p53. Rather, we propose that miR-23a and miR-27a target proapoptotic genes downstream of p53, such as PUMA, Noxa, and Bax. Our Luciferase assays confirmed that miR-23a-3p and miR-27a-3p target PUMA, Noxa, and Bax 3'UTR mRNA. Consistent with this conclusion, transfection of neurons with miR-23a-3p or miR27a-3p mimics caused significant downregulation of PUMA, Noxa, and Bax in vitro. Transfection with miR-23a-3p or miR27a-3p mimics decreased the levels of AIF-1 and cytochrome $c$ in the cytosolic fraction of etoposide-treated primary cortical neurons and reduced cleavage of caspase- 3 and PARP. $\alpha$-Fodrin undergoes both calpain-dependent cleavage $(150 / 145 \mathrm{kDa}$ fragments) and caspase-dependent cleavage (150/120 kDa fragments) after brain injury in vivo (Siman et al., 2004) and apoptosis in vitro (Cryns et al., 1996). Transfection of primary cortical neurons with miR-23a and miR-27a mimics inhibited cleavage of $\alpha$-fodrin and reduced both calpain-dependent and caspasedependent fragments.

The importance of miR-23a and miR-27a changes for TBIinduced activation of neuronal cell death pathways was demonstrated by intracerebroventricular injection of miR-23a-3p and miR-27a-3p mimics. These interventions not only attenuated injury-dependent activation of PUMA, Noxa, and Bax, but they also inhibited downstream molecular mechanisms of neuronal apoptosis in the TBI cortex. Thus, treatment with miR-23a-3p and miR-27a-3p mimics downregulates release of AIF-1 and cytochrome $c$ into the cytosol after TBI and inhibits both calpaindependent and caspase-dependent $\alpha$-fodrin cleavage. Moreover, our data demonstrate that interventions targeting selected miRs attenuate tissue loss and hippocampal neurodegeneration after TBI. Specifically, intracerebroventricular administration of miR23a-3p mimic (selected based on the strength of its molecular effects) reduced cortical lesion volume as well as neuronal cell loss in key regions of the hippocampus, such as CA2/3 and dentate gyrus, which are well-established sites of post-traumatic secondary injury (Kabadi et al., 2012).

A recent study proposes that $\mathrm{miR}-23 / \mathrm{miR}-27$ regulate the sensitivity of neurons to apoptosis in fetal hypoxia through negative modulation of Apaf-1 expression (Chen et al., 2014). Although we did not observe changes in Apaf-1 during neuronal apoptosis in vitro (Fig. 7) (Sabirzhanov et al., 2012), we have previously reported upregulation of Apaf-1 after adult TBI (Yakovlev et al., 2001). Thus, it is possible that, in addition to regulating upstream cell death molecules such as proapoptotic $\mathrm{Bcl} 2$ proteins, miR-23/ miR-27 also modulate apoptosis through regulation of downstream targets such as Apaf- 1 .

In conclusion, our results demonstrated that miR-23a and miR-27a target proapoptotic members of the Bcl-2 family Bax, PUMA, and Noxa. Downregulation of miR-23a-3p and miR$27 \mathrm{a}-3 \mathrm{p}$ after TBI or neuronal apoptosis in vitro amplifies $\mathrm{p} 53$ dependent apoptotic pathways and contributes to neuronal cell death in vivo and in vitro. Treatment with mimics of these miRs attenuates neuronal appoptosis and significantly reduced posttraumatic neurodegeneration. The distinct ability of miRs to target multiple members of a molecular pathway, as shown here, may explain the significance of their biological effects. Thus,
miR-23a and miR-27a may be potential therapeutic targets for modulation of neuronal apoptosis after TBI.

\section{References}

Chen Q, Xu J, Li L, Li H, Mao S, Zhang F, Zen K, Zhang CY, Zhang Q (2014) MicroRNA-23a/b and microRNA-27a/b suppress Apaf- 1 protein and alleviate hypoxia-induced neuronal apoptosis. Cell Death Dis 5:e1132. CrossRef Medline

Clark RSB, Chen M, Kochanek PM, Watkins SC, Jin KL, Draviam R, Nathaniel PD, Pinto R, Marion DW, Graham SH (2001) Detection of single- and double-strand DNA breaks after traumatic brain injury in rats: comparison of in situ labeling techniques using DNA polymerase I, the Klenow fragment of DNA polymerase I, and terminal deoxynucleotidyl transferase. J Neurotrauma 18:675-689. CrossRef Medline

Cryns VL, Bergeron L, Zhu H, Li H, Yuan J (1996) Specific cleavage of alpha-fodrin during Fas- and tumor necrosis factor-induced apoptosis is mediated by an interleukin-1beta-converting enzyme/Ced-3 protease distinct from the poly(ADP-ribose) polymerase protease. J Biol Chem 271:31277-31282. CrossRef Medline

Culmsee C, Mattson MP (2005) p53 in neuronal apoptosis. Biochem Biophys Res Commun 331:761-777. CrossRef Medline

Di Giovanni S, Knoblach SM, Brandoli C, Aden SA, Hoffman EP, Faden AI (2003) Gene profiling in spinal cord injury shows role of cell cycle in neuronal death. Ann Neurol 53:454-468. CrossRef Medline

Engel T, Plesnila N, Prehn JH, Henshall DC (2011) In vivo contributions of $\mathrm{BH} 3$-only proteins to neuronal death following seizures, ischemia, and traumatic brain injury. J Cereb Blood Flow Metab 31:1196-1210. CrossRef Medline

Fox GB, Fan L, Levasseur RA, Faden AI (1998) Sustained sensory/motor and cognitive deficits with neuronal apoptosis following controlled cortical impact brain injury in the mouse. J Neurotrauma 15:599-614. CrossRef Medline

Gautier L, Cope L, Bolstad BM, Irizarry RA (2004) affy-analysis of Affymetrix GeneChip data at the probe level. Bioinformatics 20:307-315. CrossRef Medline

Gentleman R (2005) Bioinformatics and computational biology solutions using R and Bioconductor. New York: Springer Science+ Business Media.

Griffiths-Jones S, Grocock RJ, van Dongen S, Bateman A, Enright AJ (2006) miRBase: microRNA sequences, targets and gene nomenclature. Nucleic Acids Res 34:D140-D144. CrossRef Medline

Guo Z, Zhou B, Liu W, Xu Y, Wu D, Yin Z, Permatasari F, Luo D (2013) MiR-23a regulates DNA damage repair and apoptosis in UVB-irradiated HaCaT cells. J Dermatol Sci 69:68-76. CrossRef Medline

Harraz MM, Eacker SM, Wang X, Dawson TM, Dawson VL (2012) MicroRNA-223 is neuroprotective by targeting glutamate receptors. Proc Natl Acad Sci U S A 109:18962-18967. CrossRef Medline

Hu Z, Yu D, Almeida-Suhett C, Tu K, Marini AM, Eiden L, Braga MF, Zhu J, Li Z (2012) Expression of miRNAs and their cooperative regulation of the pathophysiology in traumatic brain injury. PLoS One 7:e39357. CrossRef Medline

Irizarry RA, Bolstad BM, Collin F, Cope LM, Hobbs B, Speed TP (2003) Summaries of Affymetrix GeneChip probe level data. Nucleic Acids Res 31:e15. CrossRef Medline

Jeffers JR, Parganas E, Lee Y, Yang C, Wang J, Brennan J, MacLean KH, Han J, Chittenden T, Ihle JN, McKinnon PJ, Cleveland JL, Zambetti GP (2003) Puma is an essential mediator of p53-dependent and -independent apoptotic pathways. Cancer Cell 4:321-328. CrossRef Medline

Jimenez-Mateos EM, Henshall DC (2013) Epilepsy and microRNA. Neuroscience 238:218-229. CrossRef Medline

Jimenez-Mateos EM, Bray I, Sanz-Rodriguez A, Engel T, McKiernan RC, Mouri G, Tanaka K, Sano T, Saugstad JA, Simon RP, Stallings RL, Henshall DC (2011) miRNA expression profile after status epilepticus and hippocampal neuroprotection by targeting miR-132. Am J Pathol 179: 2519-2532. CrossRef Medline

Kabadi SV, Stoica BA, Hanscom M, Loane DJ, Kharebava G, Murray Ii MG, Cabatbat RM, Faden AI (2012) CR8, a selective and potent CDK inhibitor, provides neuroprotection in experimental traumatic brain injury. Neurotherapeutics 9:405-421. CrossRef Medline

Kaeser MD, Iggo RD (2002) Chromatin immunoprecipitation analysis fails to support the latency model for regulation of p53 DNA binding activity in vivo. Proc Natl Acad Sci U S A 99:95-100. CrossRef Medline 
Kole AJ, Swahari V, Hammond SM, Deshmukh M (2011) miR-29b is activated during neuronal maturation and targets $\mathrm{BH} 3$-only genes to restrict apoptosis. Genes Dev 25:125-130. CrossRef Medline

Lei P, Li Y, Chen X, Yang S, Zhang J (2009) Microarray based analysis of microRNA expression in rat cerebral cortex after traumatic brain injury. Brain Res 1284:191-201. CrossRef Medline

Le Rhun Y, Kirkland JB, Shah GM (1998) Cellular responses to DNA damage in the absence of poly(ADP-ribose) polymerase. Biochem Biophys Res Commun 245:1-10. CrossRef Medline

Li P, Nijhawan D, Budihardjo I, Srinivasula SM, Ahmad M, Alnemri ES, Wang X (1997) Cytochrome c and dATP-dependent formation of Apaf1/caspase-9 complex initiates an apoptotic protease cascade. Cell 91:479489. CrossRef Medline

Liu DZ, Tian Y, Ander BP, Xu H, Stamova BS, Zhan X, Turner RJ, Jickling G, Sharp FR (2010) Brain and blood microRNA expression profiling of ischemic stroke, intracerebral hemorrhage, and kainate seizures. J Cereb Blood Flow Metab 30:92-101. CrossRef Medline

Liu X, Kim CN, Yang J, Jemmerson R, Wang X (1996) Induction of apoptotic program in cell-free extracts: requirement for dATP and cytochrome c. Cell 86:147-157. CrossRef Medline

Livak KJ, Schmittgen TD (2001) Analysis of relative gene expression data using real-time quantitative PCR and the 2(-Delta Delta C(T)) Method. Methods 25:402-408. CrossRef Medline

Loane DJ, Pocivavsek A, Moussa CE, Thompson R, Matsuoka Y, Faden AI, Rebeck GW, Burns MP (2009) Amyloid precursor protein secretases as therapeutic targets for traumatic brain injury. Nat Med 15:377-379. CrossRef Medline

Lomonosova E, Chinnadurai G (2008) BH3-only proteins in apoptosis and beyond: an overview. Oncogene 27 [Suppl 1]:S2-S19.

Lowe SW, Schmitt EM, Smith SW, Osborne BA, Jacks T (1993) p53 is required for radiation-induced apoptosis in mouse thymocytes. Nature 362:847-849. CrossRef Medline

Nakano K, Vousden KH (2001) PUMA, a novel proapoptotic gene, is induced by p53. Mol Cell 7:683-694. CrossRef Medline

Oda E, Ohki R, Murasawa H, Nemoto J, Shibue T, Yamashita T, Tokino T, Taniguchi T, Tanaka N (2000) Noxa, a BH3-only member of the Bcl-2 family and candidate mediator of p53-induced apoptosis. Science 288: 1053-1058. CrossRef Medline

Pfaffl MW (2001) A new mathematical model for relative quantification in real-time RT-PCR. Nucleic Acids Res 29:e45. CrossRef Medline

Pietrzak M, Smith SC, Geralds JT, Hagg T, Gomes C, Hetman M (2011) Nucleolar disruption and apoptosis are distinct neuronal responses to etoposide-induced DNA damage. J Neurochem 117:1033-1046. CrossRef Medline

Redell JB, Liu Y, Dash PK (2009) Traumatic brain injury alters expression of hippocampal microRNAs: potential regulators of multiple pathophysiological processes. J Neurosci Res 87:1435-1448. CrossRef Medline

Redell JB, Zhao J, Dash PK (2011) Altered expression of miRNA-21 and its targets in the hippocampus after traumatic brain injury. J Neurosci Res 89:212-221. CrossRef Medline

Rogakou EP, Boon C, Redon C, Bonner WM (1999) Megabase chromatin domains involved in DNA double-strand breaks in vivo. J Cell Biol 146: 905-916. CrossRef Medline

Sabirzhanov B, Stoica BA, Hanscom M, Piao CS, Faden AI (2012) Overexpression of HSP70 attenuates caspase-dependent and caspaseindependent pathways and inhibits neuronal apoptosis. J Neurochem 123:542-554. CrossRef Medline

Selvamani A, Sathyan P, Miranda RC, Sohrabji F (2012) An antagomir to microRNA Let7f promotes neuroprotection in an ischemic stroke model. PLoS One 7:e32662. CrossRef Medline

Shamas-Din A, Brahmbhatt H, Leber B, Andrews DW (2011) BH3-only proteins: orchestrators of apoptosis. Biochim Biophys Acta 1813:508520. CrossRef Medline

Shieh SY, Ikeda M, Taya Y, Prives C (1997) DNA damage-induced phos- phorylation of p53 alleviates inhibition by MDM2. Cell 91:325-334. CrossRef Medline

Siegel C, Li J, Liu F, Benashski SE, McCullough LD (2011) miR-23a regulation of X-linked inhibitor of apoptosis (XIAP) contributes to sex differences in the response to cerebral ischemia. Proc Natl Acad Sci U S A 108:11662-11667. CrossRef Medline

Siman R, Baudry M, Lynch G (1984) Brain fodrin: substrate for calpain I, an endogenous calcium-activated protease. Proc Natl Acad Sci U S A 81: 3572-3576. CrossRef Medline

Siman R, McIntosh TK, Soltesz KM, Chen Z, Neumar RW, Roberts VL (2004) Proteins released from degenerating neurons are surrogate markers for acute brain damage. Neurobiol Dis 16:311-320. CrossRef Medline

Srinivasula SM, Ahmad M, Fernandes-Alnemri T, Alnemri ES (1998) Autoactivation of procaspase- 9 by Apaf-1-mediated oligomerization. Mol Cell 1:949-957. CrossRef Medline

Stappert L, Borghese L, Roese-Koerner B, Weinhold S, Koch P, Terstegge S, Uhrberg M, Wernet P, Brüstle O (2013) MicroRNA-based promotion of human neuronal differentiation and subtype specification. PLoS One 8:e59011. CrossRef Medline

Steckley D, Karajgikar M, Dale LB, Fuerth B, Swan P, Drummond-Main C, Poulter MO, Ferguson SS, Strasser A, Cregan SP (2007) Puma is a dominant regulator of oxidative stress induced Bax activation and neuronal apoptosis. J Neurosci 27:12989-12999. CrossRef Medline

Stoica BA, Faden AI (2010) Cell death mechanisms and modulation in traumatic brain injury. Neurotherapeutics 7:3-12. CrossRef Medline

Stoica BA, Movsesyan VA, Lea PM 4th, Faden AI (2003) Ceramide-induced neuronal apoptosis is associated with dephosphorylation of Akt, BAD, FKHR, GSK-3beta, and induction of the mitochondrial-dependent intrinsic caspase pathway. Mol Cell Neurosci 22:365-382. CrossRef Medline

Stoica BA, Movsesyan VA, Knoblach SM, Faden AI (2005) Ceramide induces neuronal apoptosis through mitogen-activated protein kinases and causes release of multiple mitochondrial proteins. Mol Cell Neurosci 29: 355-371. CrossRef Medline

Susin SA, Lorenzo HK, Zamzami N, Marzo I, Snow BE, Brothers GM, Mangion J, Jacotot E, Costantini P, Loeffler M, Larochette N, Goodlett DR, Aebersold R, Siderovski DP, Penninger JM, Kroemer G (1999) Molecular characterization of mitochondrial apoptosis-inducing factor. Nature 397:441-446. CrossRef Medline

Truettner JS, Alonso OF, Bramlett HM, Dietrich WD (2011) Therapeutic hypothermia alters microRNA responses to traumatic brain injury in rats. J Cereb Blood Flow Metab 31:1897-1907. CrossRef Medline

Truettner JS, Motti D, Dietrich WD (2013) MicroRNA overexpression increases cortical neuronal vulnerability to injury. Brain Res 1533:122-130. CrossRef Medline

Vousden KH (2005) Apoptosis. p53 and PUMA: a deadly duo. Science 309: 1685-1686. CrossRef Medline

Yakovlev AG, Ota K, Wang G, Movsesyan V, Bao WL, Yoshihara K, Faden AI (2001) Differential expression of apoptotic protease-activating factor-1 and caspase- 3 genes and susceptibility to apoptosis during brain development and after traumatic brain injury. J Neurosci 21:7439-7446. Medline

Yakovlev AG, Di Giovanni S, Wang G, Liu W, Stoica B, Faden AI (2004) BOK and NOXA are essential mediators of p53-dependent apoptosis. J Biol Chem 279:28367-28374. CrossRef Medline

Yan H, Xu T, Zhao H, Lee KC, Wang HY, Zhang Y (2013) Isoflurane increases neuronal cell death vulnerability by downregulating miR-214. PLoS One 8:e55276. CrossRef Medline

Yang X, Zhou Y, Peng S, Wu L, Lin HY, Wang S, Wang H (2012) Differentially expressed plasma microRNAs in premature ovarian failure patients and the potential regulatory function of mir-23a in granulosa cell apoptosis. Reproduction 144:235-244. CrossRef Medline

Ziu M, Fletcher L, Rana S, Jimenez DF, Digicaylioglu M (2011) Temporal differences in microRNA expression patterns in astrocytes and neurons after ischemic injury. PLoS One 6:e14724. CrossRef Medline 